\title{
GENERAL DIFFERENTIAL GEOMETRIES AND RELATED TOPICS*
}

\author{
A. D. MICHAL
}

I. INTRODUCTION

1. Scope of the paper. The main purpose of this paper is to give an account of the author's recent researches on differential geometry with general coordinates. $\dagger$ In these geometries the geometric space is taken to be a Hausdorff topological space, while the coordinate space is taken to be a linear topological space. Several important topics in general analysis had their inception in these differential geometric researches. Such analytical topics come under the following captions: completely integrable abstract differential equations, $\ddagger$ boundary value problems in general analysis, $\S$ general continuous group theory with abstract parameters, $\|$ abstract analytic functions, 9 the MichalPaxson differential in special linear topological spaces, ${ }^{* *}$ and the $M$-differential in linear topological spaces. $\dagger \dagger$ A detailed account, or even a brief account, of most of these purely analytical matters is, however, out of the question here.

We are convinced that the subject of general differential geometry is destined to become one of the great branches of mathematics, comparable to the present status of general (abstract) algebra and general analysis. There is still time for a whole army of young mathematicians to earn their first laurels in general differential geometry while the subject is still in its infancy.

2. Special instances and special features. The differential geometries treated are dimensionless in the sense that no dimensionality postulate (finite or infinite) is specified. The general theory thus contains as instances the classical Riemannian and non-Riemannian geometries with finite or infinite number of dimensions. An account of these instances has, however, been crowded out. The interested reader

* An address delivered before the Berkeley meeting of the Society on April 9, 1938, by invitation of the Program Committee.

$\dagger$ Michal [6-13]. The numbers refer to the entries in the bibliography at the end of the paper.

$\ddagger$ Michal and Elconin $[1,3]$.

$\S$ Michal and Hyers [1].

\|I Michal and Paxson [1, 2]; Michal and Elconin [2]; Michal, Highberg, and Taylor [1].

I Martin [1]; Michal and Martin [1]; Michal and Clifford [1]; Taylor [2-6].

** Michal and Paxson [3, 4].

$\dagger \dagger$ Michal [13]. 
will notice that the general theory presented here furnishes readily many novel results in the finite dimensional instances and especially in the infinite dimensional functional instances.

Although much of the general theory presented here has been published or is in the press, there are many results that are given here for the first time. For example, large portions of Chapters III, IV, V, and XI, and Theorem 14.3 of Chapter VI are new.

The work* on abstract normal coordinates in collaboration with D. H. Hyers is reported in Chapters VIII and IX.

\section{INFinitely many Dimensional Differential GeOMEtRies}

3. Historical remarks. Although finite dimensional as well as various infinite dimensional differential geometries furnish noteworthy instances of general differential geometries with abstract coordinates, it was the infinite dimensional instances that paved the way to the general theories. More specifically, the author's $\dagger$ researches during the period 1927-1931 on Riemannian and non-Riemannian geometries with coordinates in the space of real continuous functions of a real variable showed the need for simplifying generalizations and for a study of the foundations. Although the writing of continuous variables as indices together with the use of the integration convention $\ddagger$ for an index repeated once as a subscript and once as a superscript was helpful, the functional algebra was still too complicated to allow significant new advances. Motivated by such considerations as these, the author began his researches on general differential geometry with Banach coordinates. The turn of events in these studies was most gratifying-not only were numerous fundamental problems in the function space geometries solved automatically, but also the whole subject of differential geometry was viewed in clearer light from new vantage points.

4. An infinite dimensional Riemannian geometry. This section and the next one are not intended to be even brief accounts of differential geometries with infinite dimensions, but are merely indicative of the trend in formal outlook from the finite dimensional geometries to the infinite dimensional geometries. In my first studies $\$$ in 1927, the element of arc length $d s$ of the functional Riemannian geometry was given by

\footnotetext{
* Michal and Hyers [2-4].

$\dagger$ Michal [1-5].

$\ddagger$ For example, $\int_{a}^{b} K(x, s) y(s) d s$ is written as $K_{s}^{x} y^{s}$.

$\S$ Michal [1].
} 


$$
d s=\left(\int_{a}^{b}\left(\delta x^{\alpha}\right)^{2} d \alpha+g_{\alpha \beta}\left[x^{\sigma}\right] \delta x^{\alpha} \delta x^{\beta}\right)^{1 / 2} .
$$

For invariantive purposes, this necessitated the transformation of coordinates $\bar{x}^{\alpha}=f^{\alpha}\left[x^{\sigma}\right]$ to have Fréchet differentials of the second kind (Fredholm type)

$$
\delta \bar{x}^{\alpha}=\delta x^{\alpha}+\phi_{\beta}{ }^{\alpha}\left[x^{\sigma}\right] \delta x^{\beta} .
$$

Soon thereafter the element of arc length was taken in the more general form

$$
d s=\left(g_{\alpha}\left[x^{\sigma}\right]\left(\delta x^{\alpha}\right)^{2}+g_{\alpha \beta}\left[x^{\sigma}\right] \delta x^{\alpha} \delta x^{\beta}\right)^{1 / 2} .
$$

The transformation of coordinates was then allowed to have Fréchet differentials of the third kind:

$$
\delta \bar{x}^{\alpha}=\psi^{\alpha}\left[x^{\sigma}\right] \delta x^{\alpha}+\psi_{\beta}^{\alpha}\left[x^{\sigma}\right] \delta x^{\beta} .
$$

5. An infinite dimensional differential geometry with a linear connection.* The fundamental geometric object in the infinite dimensional Riemannian geometry with element of arc length (4.3) is the metric functional tensor with components consisting of the ordered pair of functionals $\left(g_{\alpha}\left[x^{\sigma}\right], g_{\alpha \beta}\left[x^{\sigma}\right]\right)$. The functional "Christoffel" symbols based on the metric functional tensor constitute the fundamental linear connection of the geometry.

The fundamental geometric object (not a functional tensor) in an infinite dimensional differential geometry with a functional linear connection is the functional linear connection (functional affine connection is an alternative terminology). The components of the functional linear connection consist of an ordered sequence of functionals

$$
\left(\Gamma_{\alpha \beta}^{i}\left[x^{\sigma}\right], M_{\alpha}^{i}\left[x^{\sigma}\right], N_{\alpha}^{i}\left[x^{\sigma}\right], O_{\alpha}^{i}\left[x^{\sigma}\right], P^{i}\left[x^{\sigma}\right]\right),
$$

with the property that the functional covariant differential

$$
\delta \xi^{i}\left[x^{\sigma}\right]+\Gamma_{\alpha \beta}^{i} \xi^{\alpha} \delta x^{\beta}+M_{\alpha}^{i} \xi^{\alpha} \delta x^{\alpha}+N_{\alpha}^{i} \xi^{i} \delta x^{\alpha}+O_{\alpha}^{i} \xi^{\alpha} \delta x^{i}+P^{i} \xi^{i} \delta x^{i}
$$

is a contravariant functional vector field whenever $\xi^{i}\left[x^{\sigma}\right]$ is a contravariant functional vector field.

\section{Differential IN LINEAR TOPOLOGICAL SPACES}

6. Topological spaces. A differential geometry is concerned with geometric spaces and their maps in coordinate spaces. A geometric space consists of a class of objects capable of supporting some sort of

* Michal [2-5]. 
a topology, while a coordinate space consists of a class of objects capable of supporting a differential calculus.

By a topological space we mean a Hausdorff topological space; that is, a class of undefined elements, called points, with a neighborhood topology satisfying the four well known Hausdorff postulates (Fréchet [2], Hausdorff [1]):

(1) $x \varepsilon S_{x}$.

(2) If $y \varepsilon S_{x}$, then there exists an $S_{y} \subset S_{x}$.

(3) $S_{x}^{1} S_{x}^{2} \supset S_{x}^{3}$.

(4) If $x \neq y$, then there exist $S_{x}$ and $S_{y}$ such that the set intersection $S_{x} S_{y}$ is the null set.

By a linear topological space (Kolmogoroff [1], Tychonoff [1]), we mean a topological space whose points form an abstract linear space such that the sum function $x+y$ and the product function $\alpha x$, with real variable $\alpha$, are respectively continuous functions of both variables.

As the coordinate spaces of the differential geometries to be reported on in the sequel are linear topological spaces, it is convenient in this section to give the fundamentals of a differential calculus* of functions with arguments and values in linear topological spaces. Many kinds of definitions of differentials have been given in the classical differential calculi of finite, as well as of infinite, dimensional spaces. However, the most useful definitions from the point of view of the geometrical applications are those that give the differential as a "first order approximation" to the increment.

Let $T_{1}$ and $T_{2}$ be any two linear topological spaces. It is to be observed that $T_{1}$ and $T_{2}$ are not necessarily normed metric spaces-not even normable spaces. For a necessary and sufficient condition that a linear topological space be normable see Kolmogoroff [1]. A function $l(x)$ on $\dagger T_{1}$ to $T_{2}$ is termed linear if it is additive and continuous. By the usual methods, we see that a linear function $l(x)$ on $T_{1}$ to $T_{2}$ is a homogeneous function of degree one.

7. A topological differential. We make the following definition:

DEFINITION OF $M$-DifFERENTIAL. Let $f(x)$ be a function on $S_{x_{0}}$ to $T_{2}$, where $S_{x_{0}}$ is a Hausdorff neighborhood of $x_{0} \varepsilon T_{1}$. The function $f(x)$ will

* Michal [13]. The extensions to complex linear topological spaces are made in the obvious way. For example, a linear function $l(x)$ will be an additive continuous function such that $l\left((-1)^{1 / 2} x\right)=(-1)^{1 / 2} l(x)$.

$\dagger$ By $f(x)$ on $S_{1}$ to $S_{2}$ we mean that $f(x)$ is defined throughout the set $S_{1}$ and with values contained in the set $S_{2}$ but not necessarily exhausting the whole set $S_{2}$. 
be said to be $M$-differentiable at $x=x_{0}$, and $f\left(x_{0} ; \delta x\right)$ will be called an $M$-differential of $f(x)$ at $x=x_{0}$ with increment $\delta x$, if

(1) there exists a linear function $f\left(x_{0} ; \delta x\right)$ of $\delta x$ on $T_{1}$ to $T_{2}$;

(2) there exists a function $\epsilon\left(x_{0}, x_{1}, x_{2}\right)$ with arguments in $T_{1}$ and values in $T_{2}$ such that

(2a) $\epsilon\left(x_{0}, 0, x\right)=0$ for all $x \varepsilon T_{1}$,

(2b) $\epsilon\left(x_{0}, x_{1}, \lambda x_{2}\right)=\lambda \epsilon\left(x_{0}, x_{1}, x_{2}\right)$ for all $\lambda>0$, for all $x_{1}$ in some Hausdorff neighborhood of $0 \varepsilon T_{1}$, and for all $x_{2} \varepsilon T_{1}$,

(2c) $\epsilon\left(x_{0}, x_{1}, x_{2}\right)$ is continuous in $\left(x_{1}, x_{2}\right)$ at $x_{1}=0, x_{2}=x_{2}$ for all $x_{2} \varepsilon T_{1}$;

(3) there exists some Hausdorff neighborhood $S_{0}^{\prime}$ of 0 \& $T_{1}$ such that, for all $\delta x \varepsilon S_{0}^{\prime}$, the differential $f\left(x_{0} ; \delta x\right)$ is a first order approximation to the increment $f\left(x_{0}+\delta x\right)-f\left(x_{0}\right)$ in the sense that

for all $\delta x \varepsilon S_{0}^{\prime}$

$$
f\left(x_{0}+\delta x\right)-f\left(x_{0}\right)-f\left(x_{0} ; \delta x\right)=\epsilon\left(x_{0}, \delta x, \delta x\right)
$$

THEOREM 7.1. If an $M$-differential of $f(x)$ at $x=x_{0}$ exists, then it is unique and $f(x)$ is continuous at $x=x_{0}$.

Theorem 7.2. If $f_{1}(x)$ and $f_{2}(x)$ are $M$-differentiable at $x=x_{0}$, then $f_{3}(x)=\alpha f_{1}(x)+\beta f_{2}(x)$ is $M$-differentiable at $x=x_{0}$ and

$$
f_{3}\left(x_{0} ; \delta x\right)=\alpha f_{1}\left(x_{0} ; \delta x\right)+\beta f_{2}\left(x_{0} ; \delta x\right) .
$$

THEOREM 7.3. Let $T_{3}$ be a third linear topological space. If $f(x)$ on $S_{x_{0}} \subset T_{1}$ to $T_{2}$ is $M$-differentiable at $x=x_{0}$, and if $\phi(y)$ on ${ }^{*} f\left(S_{x_{0}}\right)$ to $T_{3}$ is $M$-differentiable at $y_{0}=f\left(x_{0}\right)$, then $\psi(x)=\phi(f(x))$ is $M$-differentiable at $x=x_{0}$ and

$$
\psi\left(x_{0} ; \delta x\right)=\phi\left(f\left(x_{0}\right) ; f\left(x_{0} ; \delta x\right)\right) .
$$

Proof. There exists a Hausdorff neighborhood $V_{0}$ of $0 \varepsilon T_{2}$ such that, for all $\delta y \varepsilon V_{0}$,

$$
\phi\left(y_{0}+\delta y\right)-\phi\left(y_{0}\right)-\phi\left(y_{0} ; \delta y\right)=\epsilon_{1}\left(y_{0}, \delta y, \delta y\right) .
$$

Take $y_{0}=f\left(x_{0}\right)$. By Theorem 7.1, there exists a neighborhood $S_{0}^{\prime}$ of $0 \varepsilon T_{1}$ such that $f\left(x_{0}+\delta x\right)-f\left(x_{0}\right) \varepsilon V_{0}$ for each $\delta x \varepsilon S_{0}^{\prime}$. Take $\delta y=f\left(x_{\alpha}+\delta x\right)-f\left(x_{0}\right)$ and obtain

$$
\begin{aligned}
\phi\left(f\left(x_{0}+\delta x\right)\right)-\phi( & \left.\left(x_{0}\right)\right)-\phi\left(f\left(x_{0}\right) ; f\left(x_{0}+\delta x\right)-f\left(x_{0}\right)\right) \\
& =\epsilon_{1}\left(f\left(x_{0}\right), f\left(x_{0}+\delta x\right)-f\left(x_{0}\right), f\left(x_{0}+\delta x\right)-f\left(x_{0}\right)\right)
\end{aligned}
$$

for $\delta x \varepsilon S_{0}^{\prime}$. But, by hypothesis, there exists a neighborhood $\bar{S}_{0}$ of $0 \varepsilon T_{1}$ such that, for all $\delta x \varepsilon \bar{S}_{0}$,

$$
f\left(x_{0}+\delta x\right)-f\left(x_{0}\right)=f\left(x_{0} ; \delta x\right)+\epsilon_{2}\left(x_{0}, \delta x, \delta x\right) .
$$

\footnotetext{
* The notation $f\left(S_{x_{0}}\right)$ stands for the set of values of $f(x)$ as $x$ ranges over the set $S_{x_{0}}$.
} 
Consequently, from the definition of $\psi(x)$, the properties of the $\epsilon_{1}$ and $\epsilon_{2}$ functions, the linearity of $\phi\left(y_{0} ; \delta y\right)$, and the obvious use of the Hausdorff postulates, there exists a neighborhood $S_{0}^{\prime \prime}$ of $0 \varepsilon T_{1}$ such that, for all $\delta x \varepsilon S_{0}^{\prime \prime}$,

$$
\begin{aligned}
\psi\left(x_{0}+\delta x\right)-\psi\left(x_{0}\right)-\phi\left(f\left(x_{0}\right) ; f\left(x_{0} ; \delta x\right)\right)=\phi\left(f\left(x_{0}\right) ; \epsilon_{2}\left(x_{0}, \delta x, \delta x\right)\right) \\
+\epsilon_{1}\left(f\left(x_{0}\right), f\left(x_{0} ; \delta x\right)+\epsilon_{2}\left(x_{0}, \delta x, \delta x\right), f\left(x_{0} ; \delta x\right)+\epsilon_{2}\left(x_{0}, \delta x, \delta x\right)\right) .
\end{aligned}
$$

Define

$$
\begin{aligned}
& \epsilon_{3}\left(x_{0}, x_{1}, x_{2}\right)=\phi\left(f\left(x_{0}\right) ; \epsilon_{2}\left(x_{0}, x_{1}, x_{2}\right)\right) \\
& \quad+\epsilon_{1}\left(f\left(x_{0}\right), f\left(x_{0} ; x_{1}\right)+\epsilon_{2}\left(x_{0}, x_{1}, x_{1}\right), f\left(x_{0} ; x_{2}\right)+\epsilon_{2}\left(x_{0}, x_{1}, x_{2}\right)\right) .
\end{aligned}
$$

Clearly

$$
\epsilon_{3}\left(x_{0}, 0, x\right)=0, \epsilon_{3}\left(x_{0}, x_{1}, \lambda x_{2}\right)=\lambda \epsilon_{3}\left(x_{0}, x_{1}, x_{2}\right), \quad \text { for } \lambda>0 .
$$

The continuity of $\epsilon_{3}\left(x_{0}, x_{1}, x_{2}\right)$ in $\left(x_{1}, x_{2}\right)$ at $\left(0, x_{2}\right)$ follows without much difficulty from the continuity properties of the defining functions. Hence $\epsilon_{3}\left(x_{0}, x_{1}, x_{2}\right)$ is an admissible $\epsilon\left(x_{0}, x_{1}, x_{2}\right)$ function. The theorem follows readily on noting that $\phi\left(f\left(x_{0}\right) ; f\left(x_{0} ; \delta x\right)\right)$ is a linear function of $\delta x$.

\section{OTHER DIFFERENTIALS AND THEIR RELATION TO THE $M$-DIFFERENTIAL}

8. $G$-differential and $H M$-differential. We make the following definition:

DEFINITION OF $G$-DIFFERENTIAL. Let $f(x)$ be a function on $S_{x_{0}}$ to $T_{2}$, where $S_{x_{0}}$ is a Hausdorff neighborhood of $x_{0} \varepsilon T_{1}$. We shall say that $f(x)$ is G-differentiable at $x=x_{0}$, and $f\left(x_{0}, \delta x\right)$ is its G-differential at $x=x_{0}$ with increment $\delta x$, if, for any chosen $\delta x \varepsilon T_{1}$ :

Given any Hausdorff neighborhood $V_{0}$ of $0 \varepsilon T_{2}$, there exists $a \delta>0$ such that*

$$
\frac{f\left(x_{0}+\lambda \delta x\right)-f\left(x_{0}\right)}{\lambda} \varepsilon f\left(x_{0}, \delta x\right)+V_{0}
$$

for each $\lambda$ satisfying $0<|\lambda|<\delta$.

THEOREM 8.1. If $f(x)$ is $M$-differentiable at $x=x_{0}$, then $f(x)$ is $G$-differentiable at $x=x_{0}$, and the two differentials are equal.

DEFINITION OF $H M$-DIFFERENTIAL. Let $x_{0}$ be any chosen element of $T_{1}, S_{x_{0}}$ any chosen Hausdorff neighborhood of $x_{0}$, and $\lambda_{0}$ any chosen real

* By $f\left(x_{0}, \delta x\right)+V_{0}$ we mean the set of all elements $f\left(x_{0}, \delta x\right)+y$, as $y$ ranges over the neighborhood $V_{0}$. 
number. Let $x(\lambda)$ be a function of a real variable $\lambda$ with values in $S_{x_{0}}$ such that $x_{0}=x\left(\lambda_{0}\right)$, and such that $d x(\lambda) / d \lambda$ exists at $\lambda=\lambda_{0}$. A function $f(x)$ on $S_{x_{0}}$ to $T_{2}$ will be said to be HM-differentiable at $x=x_{0}$ with $f\left(x_{0}: \delta x\right)$ as its $H M$-differential at $x=x_{0}$, if there exists a linear function $f\left(x_{0}: \delta x\right)$ of $\delta x$ having arguments in $T_{1}$ and values in $T_{2}$ such that, for every admissible $x(\lambda)$ :

(1) $d f(x(\lambda)) / d \lambda$ exists at $\lambda=\lambda_{0}$;

(2) $d f(x(\lambda)) / d \lambda=f\left(x_{0}: d x(\lambda) / d \lambda\right)$ for $\lambda=\lambda_{0}$.

THEOREM 8.2. If $f(x)$ is $M$-differentiable at $x=x_{0}$, then $f(x)$ is HMdifferentiable at $x=x_{0}$, and the two differentials are equal.

A modified $H M$-differential is obtained if, in the definition for an $H M$-differential, $\lambda_{0}$ is always taken to be $\lambda_{0}=0$. This modified $H M$ differential is itself the abstraction of a differential in a function space studied recently by Fréchet. The reader is referred to Fréchet $[3$, p. 244].

9. Differentials in linear metric spaces. A differential calculus in complete normed linear spaces (Banach spaces) was initiated by Fréchet [1]. The differential calculi in such metric spaces have been extensively studied in recent years by many authors: Fréchet, Hildebrandt, Graves, R. S. Martin, Taylor, Elconin, Hyers, Leray, Schauder, Kerner, Highberg, Paxson, Michal, and several others. For the literature on the subject the reader is referred to the bibliographical entries for these authors.

A Banach space is a complete linear space with a topology determined by a norm $\|x\|$ that satisfies the following postulates: (1) $\|x+y\| \leqq\|x\|+\|y\| ;$; (2) $\|\alpha x\|=|\alpha|\|x\|$; (3) $\|x\| \geqq 0$ and $\|x\|=0$ if and only if $x=0$. A Banach space is then clearly a linear topological space whose Hausdorff neighborhoods $S_{x_{0}}$ are "spherical" neighborhoods $\left\|x-x_{0}\right\|<\delta$ with respect to which the generalized Cauchy criterion for convergence holds. Although a Banach space is a special kind of a linear topological space with a metric topology, it is sufficiently general to include as instances many function spaces. Moreover, a Hilbert space (real) is an infinitely dimensional separable Banach space whose norm is defined by $\|x\|=(x, x)^{1 / 2}$ in terms of a postulated symmetric bilinear inner product $(x, y)$. For such cognate subjects as linear transformations in Banach spaces and Hilbert spaces the reader is referred to Banach [1] and Stone [1].

THeOREM 9.1. If the linear topological spaces $T_{1}$ and $T_{2}$ are complete linear normed spaces (Banach spaces), and if $f(x)$ is Fréchet differentiable at $x=x_{0}$, then $f(x)$ is $M$-differentiable at $x=x_{0}$, and the two differentials are equal. 
TheOREM 9.2. If the linear topological spaces $T_{1}$ and $T_{2}$ are finite dimensional arithmetic spaces, and if $f(x)$ is differentiable at $x=x_{0}$ in the Stolz-Young-Frêchet sense, then $f(x)$ is $M$-differentiable at $x=x_{0}$, and the differentials are equal. Conversely, if $f(x)$ is $M$-differentiable at $x=x_{0}$, then it is differentiable at $x=x_{0}$ in the Stolz-Young-Fréchet sense.

10. Further topics in topological differential calculus. A slight generalization of the $M$-differential discussed in $\$ 7$ can be obtained by replacing condition (3) in the definition of an $M$-differential by the following less stringent condition:

$\left(^{\prime}\right)$ there exists some Hausdorff neighborhood $S_{0}^{\prime}$ of $0 \varepsilon T_{1}$ such that, for all $\delta x \in S_{0}{ }^{\prime}$, the differential $f\left(x_{0} ; \delta x\right)$ is a first order approximation to the increment $f\left(x_{0}+\delta x\right)-f\left(x_{0}\right)$ in the sense that

$$
f\left(x_{0}+\delta x\right)-f\left(x_{0}\right)-f\left(x_{0} ; \delta x\right) \varepsilon \Pi
$$

for all $\delta x \varepsilon S_{0}^{\prime}$, where $\Pi$ stands for the set of values of $\epsilon\left(x_{0}, \delta x, \delta x\right)$ as $\delta x$ ranges over $S_{0}^{\prime}$.

It would be interesting to develop the properties of such a modified $M$-differential.

In case $T_{1}$ is a special linear topological space and $T_{2}$ is the same space as $T_{1}$, a certain topological differential was studied by Michal and Paxson. It is still an open question whether the differentiability theorem on the composition of functions is valid for the MichalPaxson differential. If $T_{1}$, however, is further conditioned to be a Banach space, the Michal-Paxson differential reduces to a Fréchet differential and the validity of the theorem is then evident. For a systematic development of the Michal-Paxson differential, the reader is referred to Michal and Paxson [4].

\section{Differentiable manifolds With Linear topological COORDINATES}

11. Coordinate systems. It has already been remarked that a differential geometry deals with the theory of geometric spaces (Hausdorff topological spaces) and their maps in coordinate spaces (linear topological spaces). The mathematical "cameras," the mapping functions, are what we call the coordinate systems, while the "image" of a geometric point $P$ is what we call the coordinate* of $P$. The undefined elements for a differential geometry are not only the geometric

* This terminology is in slight conflict with the usage of this word in classical $n$-dimensional geometry. According to our new terminology, the one-rowed matrix $x$ of $n$ numbers $x=\left(x^{1}, x^{2}, \cdots, x^{n}\right)$ will constitute the coordinate of the point $P$. 
points and their coordinates, but also the coordinate systems and their geometrical and coordinate domains.

Let us proceed now with the more precise postulational formulation. Let $H$, the geometric space, be a Hausdorff topological space, and let $T$, the coordinate space, be a linear topological space. We postulate the existence of a class $\kappa$ of undefined coordinate systems (allowable coordinate systems), mapping functions with arguments in $H$ and values in $T$, that satisfies the following postulates:

(1) To each Hausdorff neighborhood $U$ there corresponds at least one coordinate system $x(P)$ of $\kappa$ that maps homeomorphically $U$ on some open set $O$ of $T$ (call $U$ the geometrical domain and $O$ the coordinate domain, respectively, of the coordinate system $x(P))$.

(2) All such coordinate domains $O$ are contained in some fixed open set $O_{0}$.

(3) There exists a Hausdorff neighborhood $U_{0}$ and a coordinate system $x_{0}(P)$ of $\kappa$ with $O_{0}$ as its coordinate domain.

(4) If a Hausdorff neighborhood $U_{1} \subset U$, and if $U$ is the geometrical domain of a coordinate system $x(P)$, then $x(P)$, with $U_{1}$ as a geometrical domain, is a coordinate system of $\kappa$.

(5) If $x(P)$ is a coordinate system with $U$ and $O$ as geometrical and coordinate domains, respectively, and $\bar{x}(x)$ is a homeomorphism taking $O$ into an open set $O_{1}$ of $T$, then $\bar{x}(x(P))$ is a coordinate system of $\kappa$ with $U$ as geometrical domain and $O_{1}$ as coordinate domain.

If two Hausdorff neighborhoods $U_{1}$ and $U_{2}$ of $H$ intersect, we have two mappings of their intersection on open subsets $\bar{O}_{1} \subset O_{1}$ and $\bar{O}_{2} \subset O_{2}$, respectively. This establishes a homeomorphism $\bar{x}(x)$, called a transformation of coordinates $\left(\bar{O}_{1}\right.$ and $\bar{O}_{2}$ will be called the domains of definition of $\bar{x}(x)$ and $x(\bar{x})$ respectively), that takes an open subset $\bar{O}_{1}$ of $O_{1}$ into an open subset $\bar{O}_{2}$ of $O_{2}$. Conversely, ${ }^{*}$ if $\bar{x}=\psi(x)$ is a homeomorphism taking an open set $O_{1} \subset O_{0}$ into an open set $O_{2} \subset O_{0}$, then there exist two coordinate systems $x(P)$ and $\bar{x}(P)$ of $\kappa$ with coordinate domains $O_{1}^{\prime} \subset O_{1}$ and $O_{2}^{\prime} \subset O_{2}$, respectively, such that $\bar{x}=\psi(x)$ on $O_{1}^{\prime}$ to $O_{2}^{\prime}$ is a transformation of coordinates from the system $x(P)$ to the system $\bar{x}(P)$.

It is possible now to define a scalar field, a geometrical object with components. A scalar field is a numerically valued function $f(P)$, defined on a subset of the geometrical space, such that its components in two coordinate systems $x\left(P_{1}\right)$ and $\bar{x}\left(P_{2}\right)$,

$$
\alpha(x)=f\left(P_{1}(x)\right), \quad \bar{\alpha}(\bar{x})=f\left(P_{2}(\bar{x})\right),
$$

* If the linear topological space $T$ is specialized to a Banach space, see Michal and Hyers [3], and Michal and Paxson [2]. 
are related by $\bar{\alpha}(\bar{x})=\alpha(x)$ throughout the intersection of the geometrical domains of $x\left(P_{1}\right)$ and $\bar{x}\left(P_{2}\right)$.

One can obviously extend the terminology and consider scalar fields for which the values of $f(P)$ are in any class of objects.

12. Differentiable manifolds and contravariant vectors. We shall now assume that each transformation of coordinates $\bar{x}=\bar{x}(x)$, and its inverse $x=x(\bar{x})$, possess first $M$-differentials $\bar{x}(x ; \delta x)$, continuous in $x$, and $x(\bar{x} ; \delta \bar{x})$, continuous in $\bar{x}$, throughout their respective domains of definition. A geometric space that satisfies the postulates of $\$ 11$, and such that the transformations of coordinates satisfy the above conditions, will be called a 1-differentiable manifold (with linear topological coordinates).

It can be shown that $\bar{x}(x ; \lambda)$ is a solvable linear function of $\lambda$ with $x(\bar{x}(x) ; \mu)$ as inverse for each $x$ in the domain of definition of $\bar{x}(x)$.

Contravariant vectors and contravariant vector field (c.v.f.) can now be defined for a 1-differentiable manifold. A contravariant vector (differential) is a geometric object with a component in each coordinate system such that, in the intersection of two Hausdorff neighborhoods, the characteristic law of transformation relating its components in the two coordinate systems is* $\bar{\xi}=\bar{x}(x ; \xi)$.

A contravariant vector field can now be defined in the obvious way. The characteristic law under which its components transform is given by

$$
\bar{\xi}(\bar{x})=\bar{x}(x ; \xi(x)) .
$$

\section{DifFERENTIABLE MANIFOLD WITH A LINEAR CONNECTION}

13. Linear connection. A geometric object called a linear connection plays a fundamental role in finite dimensional differential geometry as well as in the author's infinite dimensional geometries. It is natural, therefore, at this early stage of the development of our subject to add additional restrictions on our coordinate systems and transformations so as to make possible the definition of a linear connection in our present more general situation. Although it is possible to go far formally without specializing the linear topological coordinate space, and although actually one can prove many theorems with the aid of long cumbersome restrictions - automatically satisfied in the special case of a Banach space-it seems more feasible in the present exposition to restrict ourselves to the more special linear metric coordinate space. So from now on the linear topological coordinate space $T$ will be required to be a Banach space. We shall also follow from now

\footnotetext{
* Michal [8].
} 
on the historical development and require all differentials to be Fréchet differentials.

Let us consider then a 1-differentiable manifold (with Banach coordinates), and let us assume that the second successive differential $\bar{x}\left(x ; \delta_{1} x ; \delta_{2} x\right)$ exists continuously in $x$ throughout the domain of definition of each transformation of coordinates $\bar{x}(x)$. We shall call such a manifold a 2-differentiable manifold. An m-differentiable manifold is defined in the obvious manner.

If $H$ is a 2-differentiable manifold, then any transformation of coordinates $\bar{x}(x)$, and its inverse $x(\bar{x})$, have first and second differentials throughout their domains of definition. A geometrical object with components $\Gamma\left(x, \xi_{1}, \xi_{2}\right)$, bilinear in two contravariant vectors $\xi_{1}$ and $\xi_{2}$, will be called a linear connection* if, under a transformation of coordinates, the components $\Gamma\left(x, \xi_{1}, \xi_{2}\right)$ undergo the transformation

$$
\bar{\Gamma}\left(\bar{x}, \bar{\xi}_{1}, \bar{\xi}_{2}\right)=\bar{x}\left(x ; \Gamma\left(x, \xi_{1}, \xi_{2}\right)\right)+\bar{x}\left(x ; x\left(\bar{x} ; \bar{\xi}_{1} ; \bar{\xi}_{2}\right)\right) .
$$

This transformation law can also be written in the following equivalent form

$$
\bar{\Gamma}\left(\bar{x}, \bar{\xi}_{1}, \bar{\xi}_{2}\right)=\bar{x}\left(x ; \Gamma\left(x, \xi_{1}, \xi_{2}\right)\right)-\bar{x}\left(x ; \xi_{1} ; \xi_{2}\right) .
$$

14. Covariant differential of a contravariant vector field. If $\alpha(x)$ is a scalar field, then the differential $\alpha(x ; \delta x)$ is a scalar field valued linear form in the contravariant vector $\delta x$, that is, under a transformation of coordinates

$$
\bar{\alpha}(\bar{x} ; \delta \bar{x})=\alpha(x ; \delta x) .
$$

On the other hand, if $\xi(x)$ is a differentiable contravariant vector field, it is not true in general that the differential $\xi(x ; \delta x)$ is a c.v.f. (contravariant vector field) valued linear form in a contravariant vector $\delta x$. However, if the geometric space $H$ is at least a 2-manifold with a linear connection $\Gamma\left(x, \xi_{1}, \xi_{2}\right)$, then, for every differentiable contravariant vector field $\xi(x)$, the form $\xi(x \mid \delta x)$ defined by

$$
\xi(x \mid \delta x)=\xi(x ; \delta x)+\Gamma(x, \xi(x), \delta x)
$$

is a c.v.f. valued linear form in the contravariant vector $\delta x$. We shall call $\xi(x \mid \delta x)$ the covariant differential $\dagger$ of the contravariant vector field $\xi(x)$.

Since $\xi(x \mid \delta x)$ is a c.v.f. valued linear form in a contravariant vector $\delta x$, it is clear that the successive covariant differentials of a c.v.f.

* Michal $[8,9]$.

$\dagger$ Michal [8]. 
$\xi(x)$ can be made to depend on the covariant differential of a c.v.f. valued multilinear form in several contravariant vectors.

THEOREM 14.1. Let $\Gamma(x, \xi, \delta x)$ be a linear connection in a 2-differentiable manifold and $F\left(x, \xi_{1}, \xi_{2}, \cdots, \xi_{n}\right)$ a function with the following properties:

(i) $F$ is a c.v.f. valued multilinear form in the $n$ arbitrary contravariant vectors $\xi_{1}, \ldots, \xi_{n}$;

(ii) the partial differential $F\left(x, \xi_{1}, \xi_{2}, \cdots, \xi_{n} ; \delta x\right)$ exists continuously in $x$ throughout the coordinate domain of the coordinate system $x(P)$.

Then the function $F\left(x, \xi_{1}, \xi_{2}, \cdots, \xi_{n} \mid \delta x\right)$ defined by the equation

$$
\begin{aligned}
F\left(x, \xi_{1}, \xi_{2}, \cdots, \xi_{n} \mid \delta x\right)=F\left(x, \xi_{1}, \xi_{2}, \cdots, \xi_{n} ; \delta x\right) \\
\quad-\sum_{i=1}^{n} F\left(x, \xi_{1}, \cdots, \xi_{i-1}, \Gamma\left(x, \xi_{i}, \delta x\right), \xi_{i+1}, \cdots, \xi_{n}\right) \\
+\Gamma\left(x, F\left(x, \xi_{1}, \xi_{2}, \cdots, \xi_{n}\right), \delta x\right)
\end{aligned}
$$

is a c.v.f. valued multilinear form in $\xi_{1}, \xi_{2}, \cdots, \xi_{n}, \delta x$. We shall call $F\left(x, \xi_{1}, \xi_{2}, \cdots, \xi_{n} \mid \delta x\right)$ the covariant differential ${ }^{*}$ of $F\left(x, \xi_{1}, \xi_{2}, \cdots, \xi_{n}\right)$.

If the geometrical space is a 3-differentiable manifold with a linear connection $\Gamma\left(x, \xi_{1}, \xi_{2}\right)$, then the requirement that the differential $\Gamma\left(x, \xi_{1}, \xi_{2} ; \delta x\right)$ exist continuously in $x$ persists under a transformation of coordinates. On applying the preceding theorem one can prove the following theorem.

THEOREM 14.2. If the geometrical space $H$ is a 3-differentiable manifold with a linear connection $\Gamma\left(x, \xi_{1}, \xi_{2}\right)$, and if the differential $\Gamma\left(x, \xi_{1}, \xi_{2} ; \delta x\right)$ exists continuously in $x$, then for every c.v.f. $\xi(x)$ with continuous second differential $\xi\left(x ; \delta_{1} x ; \delta_{2} x\right)$ in $x$ the following commutation formula holds:

$$
\begin{aligned}
\xi\left(x\left|\delta_{1} x\right| \delta_{2} x\right)-\xi\left(x\left|\delta_{2} x\right| \delta_{1} x\right)= & B\left(x, \xi(x), \delta_{1} x, \delta_{2} x\right) \\
& -2 \xi\left(x \mid \Omega\left(x, \delta_{1} x, \delta_{2} x\right)\right),
\end{aligned}
$$

where $B\left(x, \xi, \delta_{1} x, \delta_{2} x\right)$, called the curvature form, is defined by

$$
\begin{aligned}
B\left(x, \xi, \delta_{1} x, \delta_{2} x\right) & =\Gamma\left(x, \xi, \delta_{1} x ; \delta_{2} x\right)-\Gamma\left(x, \xi, \delta_{2} x ; \delta_{1} x\right) \\
+ & \Gamma\left(x, \Gamma\left(x, \xi, \delta_{1} x\right), \delta_{2} x\right)-\Gamma\left(x, \Gamma\left(x, \xi, \delta_{2} x\right), \delta_{1} x\right),
\end{aligned}
$$

and where $\Omega\left(x, \delta_{1} x, \delta_{2} x\right)$, called the torsion form, is defined by

$$
\Omega\left(x, \delta_{1} x, \delta_{2} x\right)=\left\{\Gamma\left(x, \delta_{1} x, \delta_{2} x\right)-\Gamma\left(x, \delta_{2} x, \delta_{1} x\right)\right\} / 2 .
$$

\footnotetext{
* Michal [8].
} 
The curvature form is a c.v.f. valued trilinear form, while the torsion form is a c.v.f. valued bilinear form.

With evident restrictions on the geometrical space $H$ and the linear connection $\Gamma\left(x, \xi_{1}, \xi_{2}\right)$, it is possible to consider the successive covariant differentials of the curvature form $B\left(x, \xi, \delta_{1} x, \delta_{2} x\right)$.

Besides the obvious skew-symmetric and cyclic identities* satisfied by the curvature form based on a symmetric linear connection, one can show that the following "Bianchi" identity holds for a symmetric linear connection:

$$
B\left(x, \xi, \xi_{1}, \xi_{2} \mid \xi_{3}\right)+B\left(x, \xi, \xi_{3}, \xi_{1} \mid \xi_{2}\right)+B\left(x, \xi, \xi_{2}, \xi_{3} \mid \xi_{1}\right)=0 .
$$

An indirect but elegant proof of (14.6) can be given with the aid of the properties of abstract normal coordinates. See $\$ 17$. This method of proof requires, however, additional restrictions on the space $H$ and its linear connection.

A differentiable manifold of interest is one with a fundamental geometric object consisting of a Banach scalar valued linear form $F(x, \xi)$ in a contravariant vector $\xi$. If we assume that $F(x, \xi)$ is continuously differentiable in $x$ and is a solvable linear function of $\xi$ with $F^{\prime}(x, \lambda)$ as inverse function, then $F^{\prime}(x, \lambda)$ is a c.v.f. valued linear form in a Banach scalar $\lambda$, and $\Gamma\left(x, \xi_{1}, \xi_{2}\right)$, defined by either one of the two equal expressions

$$
\Gamma\left(x, \xi_{1}, \xi_{2}\right)=-F^{\prime}\left(x, F\left(x, \xi_{1}\right) ; \xi_{2}\right)=F^{\prime}\left(x, F\left(x, \xi_{1} ; \xi_{2}\right)\right),
$$

is a linear connection with a curvature form* $B\left(x, \xi_{1}, \xi_{2}, \xi_{3}\right)=0$.

A special case of great interest is furnished by the group scalar form $F(x, \xi)$. We call $F(x, \xi)$ a group scalar form $\dagger$ if, in addition to the above restrictions, it satisfies the abstract differential equation $\ddagger$

$$
F\left(x, \xi_{1} ; \xi_{2}\right)-F\left(x, \xi_{2} ; \xi_{1}\right)=C\left(F\left(x, \xi_{1}\right), F\left(x, \xi_{2}\right)\right),
$$

where $C(\lambda, \mu)$, called a structural form, is a Banach scalar valued bilinear form in two Banach scalar variables $\lambda$ and $\mu$, that satisfies the identities $\ddagger$

$$
\begin{gathered}
C(\lambda, \mu)=-C(\mu, \lambda), \\
C(C(\lambda, \mu), \nu)+C(C(\nu, \lambda), \mu)+C(C(\mu, \nu), \lambda)=0 .
\end{gathered}
$$

THEOREM 14.3. If $F(x, \xi)$ is a group scalar form with $F^{\prime}(x, \lambda)$ as inverse function and $\Gamma\left(x, \xi_{1}, \xi_{2}\right)$ is the linear connection (14.7), then the curvature form $B_{S}\left(x, \xi_{1}, \xi_{2}, \xi_{3}\right)$ based on the symmetric linear connection

* Michal [7].

$\dagger$ The existence of a group scalar form for a differentiable group manifold with Banach coordinates is shown in Michal-Paxson [1, 2]. See also Michal-Elconin [2].

$\ddagger$ Michal-Elconin [2]. 


$$
S\left(x, \xi_{1}, \xi_{2}\right)=\left\{\Gamma\left(x, \xi_{1}, \xi_{2}\right)+\Gamma\left(x, \xi_{2}, \xi_{1}\right)\right\} / 2
$$

becomes the elegant expression

$$
B_{S}\left(x, \xi_{1}, \xi_{2}, \xi_{3}\right)=F^{\prime}\left(x, C\left(C\left(\lambda_{2}, \lambda_{3}\right), \lambda_{1}\right)\right) / 4,
$$

where $\lambda_{i}=F\left(x, \xi_{i}\right),(i=1,2,3)$, and $C(\lambda, \mu)$ is the structural form. The covariant differential of $B_{S}\left(x, \xi_{1}, \xi_{2}, \xi_{3}\right)$ based on $S\left(x, \xi_{1}, \xi_{2}\right)$ vanishes:

$$
B_{S}\left(x, \xi_{1}, \xi_{2}, \xi_{3} \mid \xi_{4}\right)=0 .
$$

VII. Parallel displacement and auto parallel curves

15. Parallel displacement of a vector field along a curve. If $x=x(t)$ is the coordinate equation of a curve $C$ in a 2-differentiable manifold with a linear connection, then the defining equations of parallelism of a c.v.f. $\xi(x)$ along the given curve $C$ are*

$$
\frac{d \xi}{d t}+\Gamma\left(x, \xi, \frac{d x}{d t}\right)=\alpha(t) \xi(x),
$$

where $\alpha(t)$ is an arbitrarily chosen numerically valued scalar field. If we then eliminate $\alpha(t)$ in (15.1), the equations of parallelism take the equivalent form

$$
l(\xi)\left\{\frac{d \xi}{d t}+\Gamma\left(x, \xi, \frac{d x}{d t}\right)\right\}=l\left(\frac{d \xi}{d t}+\Gamma\left(x, \xi, \frac{d x}{d t}\right)\right) \xi(x),
$$

where $l(\xi)$ is an arbitrary scalar valued linear form in a contravariant vector $\xi$.

If there exists a scalar valued bilinear inner product $\left[\xi_{1}, \xi_{2}\right]$ in the contravariant vectors $\xi_{1}$ and $\xi_{2}$, and if $\left[\xi_{1}, \xi_{2}\right]$ is definite in each variable-that is, $\left[\xi_{1}, \xi_{2}\right]=0$ for all $\xi_{1}$ implies $\xi_{2}=0$, and $\left[\xi_{1}, \xi_{2}\right]=0$ for all $\xi_{2}$ implies $\xi_{1}=0$-then the equations of parallel displacement (15.1) can be written

$$
[\xi, L]\left\{\frac{d \xi}{d t}+\Gamma\left(x, \xi, \frac{d x}{d t}\right)\right\}=\left[\frac{d \xi}{d t}+\Gamma\left(x, \xi, \frac{d x}{d t}\right), L\right] \xi
$$

for all $L$.

16. Differential equations of paths. Amongst the curves of special interest in a 2-differentiable manifold are the autoparallel curves, or paths. A path is a curve with the property that its "tangent" vector $d x / d t$ forms a parallel vector field along the curve with respect to the curve.* It follows immediately, therefore, from the preceding section

* Michal $[7,10]$. 
that a path satisfies the differential equation

and hence

$$
\frac{d^{2} x}{d t^{2}}+\Gamma\left(x, \frac{d x}{d t}, \frac{d x}{d t}\right)=\alpha(t) \frac{d x}{d t},
$$

$$
\begin{aligned}
l\left(\frac{d x}{d t}\right)\left\{\frac{d^{2} x}{d t^{2}}+\Gamma\left(x, \frac{d x}{d t}, \frac{d x}{d t}\right)\right\} & \\
& =l\left(\frac{d^{2} x}{d t^{2}}+\Gamma\left(x, \frac{d x}{d t}, \frac{d x}{d t}\right)\right) \frac{d x}{d t},
\end{aligned}
$$

where $l(\xi)$ is an arbitrary scalar valued linear form in a contravariant vector $\xi$. Again, if there exists a scalar valued bilinear inner product $[\xi, L]$ that is definite in each variable, a path satisfies the differential equation

$$
\begin{aligned}
{\left[\frac{d x}{d t}, L\right]\left\{\frac{d^{2} x}{d t^{2}}+\Gamma\left(x, \frac{d x}{d t},\right.\right.} & \left.\left.\frac{d x}{d t}\right)\right\} \\
& =\left[\frac{d^{2} x}{d t^{2}}+\Gamma\left(x, \frac{d x}{d t}, \frac{d x}{d t}\right), L\right] \frac{d x}{d t}
\end{aligned}
$$

for all $L$.

There always exists a parameter $s$, called an affine parameter, such that a path satisfies the differential equation

$$
\frac{d^{2} x}{d s^{2}}+\Gamma\left(x, \frac{d x}{d s}, \frac{d x}{d s}\right)=0 .
$$

Along a path an affine parameter is determined to within an affine transformation.

The question of the existence of a path passing through two given points was discussed by Michal and Hyers [1]. In this paper two point boundary value problems for abstract differential equations (16.4) were studied. For details see the Michal-Hyers paper just cited.

\section{Abstract NORMal COORDinates}

17. Existence of normal coordinates. By a normal coordinate system $y(P)$ with center $P_{0}$ we mean a coordinate system $y(P)$ with the property that, in the neighborhood of a point $P_{0}$ with coordinate $y=0$ corresponding to the value $s=0$ of the affine parameter, the equation of a path through $P_{0}$ takes the simple form

$$
y=s \alpha,
$$

where $\alpha$ is a Banach element. To prove the existence of normal co- 
ordinate systems in a differentiable manifold with a symmetric linear connection, it seems necessary to make some additional restrictions on the differentiable manifold and on the symmetric linear connection. The first study of abstract normal coordinates was made by Michal and Hyers in Michal-Hyers [2, 3]. The following fundamental existence theorem was proved.*

TheOREM 17.1. Let $\Sigma$ be the coordinate domain of an allowable $K^{(m)}$ coordinate system $x(P)$, and let the linear connection $\Gamma\left(x, \xi_{1}, \xi_{2}\right)$ be of class $C^{(n)}$ locally uniformly on $\Sigma$, subject to the restriction $m \geqq n+2$. Then corresponding to each point $q \varepsilon \Sigma$ there is a constant $c>0$ and $a$ function $h(p, x)$, of class $C^{(n)}$ uniformly on $E^{2}\left((q)_{2 c}\right)$, such that for any choice of $p$ in $(q)_{c}$ the transformation $y=h(p, x)$ is of class $K^{(n)}$ for $x \varepsilon(p)_{c}$ and defines a normal coordinate system $y(P)$ with center $P_{0}(p)$.

The preceding theorem shows that there exist normal coordinate systems in a special $m$-differentiable manifold with a symmetric linear connection. The coordinate systems, called here allowable $K^{(m)}$ coordinate systems, of the class $\kappa$ (see $\left.\$ 11\right)$ require that the transformations of coordinates are of class $K^{(m)}$. See Michal-Hyers [3] for the definition of class $K^{(m)}$ and other definitions not given explicitly here.

The following two theorems, stated and proved in Michal-Hyers [3], show clearly the importance and usefulness of normal coordinates in general differential geometry. We use a dagger $\dagger$ to denote evaluation in a normal coordinate system.

TheOREM 17.2. For $y$ in the coordinate domain of the normal coordinate system $y(P)$,

$$
+\Gamma(y, y, y)=0,
$$

while

$$
+\Gamma(0, \lambda, \lambda)=0
$$

for all $\lambda$ in the Banach coordinate space $T$.

TheOREM 17.3. Let $x(P)$ and $\bar{x}(P)$ be two allowable $K^{(n+2)}$ coordinate systems $(n \geqq 2)$ whose geometric domains have a point $P_{0}$ in common, and let the linear connection $\Gamma\left(x, \xi_{1}, \xi_{2}\right)$ be of class $C^{(n)}$ locally uniformly in the coordinate domain of $x(P)$. Suppose that $y(P)$ and $\bar{y}(P)$ are the normal coordinate systems determined by the coordinate systems $x(P)$ and $\bar{x}(P)$, respectively, and with the same point $P_{0}$ of $H$ as center. Then there exist two open subsets $S_{y}$ and $S_{\bar{y}}$ of the coordinate domains of $y(P)$ and $\bar{y}(P)$, respectively, such that

\footnotetext{
* Michal-Hyers [3].
} 
(1) 0 \& $S_{y}, 0 \varepsilon S_{\bar{y}}$;

(2) the linear coordinate transformation

$$
\bar{y}=\bar{x}(p ; y),
$$

where $p=x\left(P_{0}\right)$,

takes $S_{y}$ into $S_{\bar{y}}$; that is, normal coordinates undergo a linear transformation under a general transformation of the determining coordinates.

18. Differential invariants. The actual use of normal coordinate methods often requires the explicit formulas giving the differentials of the transformation $y=h(p, x)$ to normal coordinates in terms of the linear connection and its differentials. For simplicity of notation define $\mu(y)$ and its inverse $\nu(x)$ by

$$
\mu(y)=f(p, y), \quad \nu(x)=h(p, x) .
$$

Under the restrictions of Theorem 17.1 one can show that

$$
\begin{aligned}
\mu(0 ; \delta y) & =\delta y, \\
\mu\left(0 ; \delta_{1} y ; \delta_{2} y ; \cdots ; \delta_{r} y\right) & =-\Gamma_{r}\left(p, \delta_{1} y, \cdots, \delta_{r} y\right), 2 \leqq r \leqq n,
\end{aligned}
$$

where $\Gamma_{r}\left(x, \xi_{1}, \xi_{2}, \cdots, \xi_{r}\right)$ is the polar of the homogeneous polynomial $H_{r}(\xi)$ defined by

$$
\begin{aligned}
& H_{r}(\xi)=\Gamma_{r-1}(x, \xi, \cdots, \xi ; \xi)-\left\{\Gamma_{r-1}(x, \Gamma(x, \xi, \xi), \xi, \cdots, \xi)\right. \\
&\left.+\cdots+\Gamma_{r-1}(x, \xi, \cdots, \xi, \Gamma(x, \xi, \xi))\right\}
\end{aligned}
$$

in terms of lower order $\Gamma$ 's. There are similar formulas for the differentials of $\nu(x)$ evaluated at $x=p$. For further results and details see Michal-Hyers [3].

The covariant differential of a scalar field valued or c.v.f. valued multilinear form in several contravariant vectors obviously retains its form as a functional of the multilinear form and its differentials and of the linear connection and its differentials. This is what we mean when we say that the covariant differential is a simultaneous differential invariant of the multilinear form and the linear connection. The first covariant differential is a first order simultaneous differential invariant, while the successive covariant differentials are simultaneous differential invariants of higher order.

There are other higher order simultaneous differential invariants, called extensions of the multilinear form, that are symmetric in the new increments. This is to be contrasted to the asymmetry of successive covariant differentials (see, for example, formula (14.3)). These extensions of multilinear forms require the geometric space to be of a more restricted character in that their very definitions are in terms of normal coordinates. For example, the $r$ th extension of 
$F\left(x, \xi_{1}, \xi_{2}, \cdots, \xi_{i}\right)$ is defined in normal coordinates $y$ by

$$
\dagger F\left(0, \uparrow \xi_{1}, \uparrow \xi_{2}, \cdots, \uparrow \xi_{i} ; \delta_{1} y ; \delta_{2} y ; \cdots ; \delta_{r} y\right) \text {. }
$$

The first extension, if it exists, coincides with the first covariant differential. However, it must be borne in mind that the first covariant differential may exist and yet not be the first extension.

Similar methods can be used to define a fundamental set of differential invariants of a linear connection. Such differential invariants of the linear connection we call normal vector forms. It is clear from their definition that the normal vector forms are c.v.f. valued multilinear forms in several contravariant vectors. If

$$
A_{1}\left(x, \xi_{1}, \xi_{2}, \xi_{3}\right), A_{2}\left(x, \xi_{1}, \xi_{2}, \xi_{3}, \xi_{4}\right), \cdots, A_{r}\left(x, \xi_{1}, \xi_{2}, \cdots, \xi_{r+2}\right)
$$

are the first $r$ normal vector forms, then every differential invariant depending on a symmetric linear connection and its differentials up to the rth order can, by a very simple replacement process, be written as a functional of the first $r$ normal vector forms. This is the reason why we called the set of normal vector forms a fundamental set of differential invariants. For further details we refer the reader to the MichalHyers [3] paper, and especially to $\S \S 5,6$, and 7 of that paper. The explicit expression of the normal vector forms in terms of the symmetric linear connection and its differentials can be calculated readily with the aid of formulas (18.2) and the corresponding formulas for the differentials of $\nu(x)$.

\section{Covariant vectors AND A SPECIAL Class of NORMAL COORDINATES}

19. Covariant vectors. The geometric objects studied so far have been scalars, scalar fields, contravariant vectors, contravariant vector fields, linear connections, scalar field, and c.v.f. valued multilinear forms in one or several contravariant vectors. The concept of a covariant vector, however, has not been used explicitly in any essential manner. There is a certain amount of arbitrariness in the choice of a definition for a covariant vector. We shall make a definition that seems natural, although not the most general, for some later developments that we now have in mind. To make this definition, we must restrict the Banach coordinate space $T$ by postulating independently* of the Banach norm $\|x\|$ of $T$ a bilinear inner product $[x, y]$ on $T^{2}$ to the real numbers with the following properties:

(1) If $[x, y]=0$ for all $x \varepsilon T$, then $y=0$.

(2) If $[x, y]=0$ for all $y \varepsilon T$, then $x=0$. [1].

* Rotations in such Banach spaces have been studied in Michal-Highberg-Taylor 
We shall use the symbol $E$ to designate such a space $T$.

Unlike the situation in Hilbert space, the adjoint of a linear transformation in $E$ is not necessarily defined throughout $E$, even though it always exists. $\ddagger$ This remark motivates then the following definition. A function $T^{*}(y)$ with argument and values in $E$ will be said to be an adjoint (transformation) of a linear transformation $T(x)$ on $E$ to $E$ if

(1) $T^{*}(y)$ is linear throughout $E$;

(2) $[T(x), y]=\left[x, T^{*}(y)\right]$ for all $x, y \varepsilon E$.

Clearly an adjoint, if it exists, is unique (within the = relation of $E$ ). Some of the elementary properties of adjoint transformations are readily proved. See Michal [9], Michal-Hyers [4], as well as MichalHighberg-Taylor [1].

If then the adjoint $x^{*}(\bar{x} ; \eta)$ of the differential $x(\bar{x} ; \delta \bar{x})$ exists throughout the domain of definition of the transformation of coordinates $x=x(\bar{x})$, the transformation law of the components of a covariant vector $\S \bar{\eta}=x^{*}(\bar{x} ; \eta)$ is well defined.

In order to treat differential invariants involving both covariant and contravariant vectors, it is clear that the theory in Chapter VIII on normal coordinates must be considerably modified and extended. One of the most difficult new problems, though trivial when the coordinate space $E$ is finite dimensional, is to prove the existence and differentiability of the adjoints of the differentials of the transformation to normal coordinates.

20. Some fundamental existence theorems. The following existence theorem is of interest in itself, aside from its use in general differential geometry.

Theorem 20.1. Let $X$ be a neighborhood of a point $x_{0}$ of the space $E$ and let $\Gamma(x, \xi, \eta)$ be a function on $X E^{2}$ to $E$, bilinear and symmetric in $\xi, \eta$ and satisfying the following conditions:

(i) $\Gamma(x, \xi, \eta)$ is of class $C^{(n)}$ uniformly on $X E^{2}\left((0)_{1}\right)$.

(ii) The adjoint $\Gamma_{(2)}^{*}(x, \xi, \eta)$ exists and is of class $C^{(n)}$ uniformly on $X E^{2}\left((0)_{1}\right)$.

(iii) The adjoint $\Gamma_{(4)}^{*}(x, \xi, \eta ; \lambda)$ exists and is of class $C^{(n-1)}$ uniformly on $X E^{3}\left((0)_{1}\right)$.

Let $Y_{0} X_{0}$ be the $\xi, p$ range of definition of the solution $\mu(s \xi, p)$ (known to exist by Theorem 1.1 of Michal-Hyers [3]) of the differential system

$$
\frac{d^{2} x}{d s^{2}}+\Gamma\left(x, \frac{d x}{d s}, \frac{d x}{d s}\right)=0, \quad x(0)=p, \quad\left(\frac{d x}{d s}\right)_{0}=\xi .
$$

$\ddagger$ Michal-Highberg-Taylor [1].

$\S$ Michal [9]. 
Then the "adjoint differential system"

$$
\begin{gathered}
\frac{d^{2} z^{*}}{d s^{2}}+z^{*}\left(s, \Gamma_{(4)}^{*}\left(\mu, \mu^{\prime}, \mu^{\prime} ; \lambda\right)\right) \\
+2\left[\frac{d}{d \sigma} z^{*}\left(\sigma, \Gamma_{(2)}^{*}\left(\mu, \lambda, \mu^{\prime}\right)\right)\right]_{\sigma=8}=0, \\
z^{*}(0, \lambda)=\lambda,\left(\frac{d z^{*}(s, \lambda)}{d s}\right)_{0}=-\Gamma_{(2)}^{*}(p, \lambda, \xi)
\end{gathered}
$$

has a unique solution $z^{*}=\psi(s, \xi, p, \lambda)$, which is of class $C^{(n-1)}$ in $\xi, p, \lambda$ uniformly on $Y_{0} X_{0} E\left((0)_{1}\right)$ for each $s$ in $I$, where $I$ is the interval $0 \leqq s \leqq 1$. Furthermore, the adjoint $\mu_{(3)}^{*}(s \xi, p ; \lambda)$ of the solution $\mu(s \xi, p ; \lambda)$ of the differential system

$$
\begin{gathered}
\frac{d^{2} z}{d s^{2}}+\Gamma\left(\mu, \mu^{\prime}, \mu^{\prime} ; z\right)+2 \Gamma\left(\mu, \frac{d z}{d s}, \mu^{\prime}\right)=0, \\
z(0, \lambda)=\lambda,\left(\frac{d z(s, \lambda)}{d s}\right)_{s=0}=-\Gamma(p, \lambda, \xi),
\end{gathered}
$$

where $\mu^{\prime}=d \mu(s \xi, p) / d s$, exists and is equal to $\psi(s, \xi, p, \lambda)$.

Let now the mappings of neighborhoods of the geometric space $H$ and the postulates on coordinate systems be taken as in Chapter VIII, with the general Banach space replaced by the space $E$, and transformations of class $K^{(m)}$ replaced by transformations of class $k^{(m)}$. For the definition of transformations of class $k^{(m)}$ the reader is referred to Michal-Hyers $[2,4]$. Let further $x(P)$ be some allowable $k^{(n+2)}$ coordinate system, and assume that, for any point $x$ of the coordinate domain of $x(P)$, the linear connection $\Gamma\left(x, \xi_{1}, \xi_{2}\right)$ satisfies the following conditions:

I. $\Gamma\left(x, \xi_{1}, \xi_{2}\right)$ is a symmetric bilinear function of $\xi_{1}$ and $\xi_{2}$.

II. $\Gamma\left(x, \xi_{1}, \xi_{2}\right)$ is of class $C^{(n)}$ locally uniformly at $x$.

III. The adjoint $\Gamma_{(2)}^{*}\left(x, \xi_{1}, \xi_{2}\right)$ exists and is of class $C^{(n)}$ locally uniformly at $x$.

IV. The adjoint $\Gamma_{(4)}^{*}\left(x, \xi_{1}, \xi_{2} ; \lambda\right)$ exists and is of class $C^{(n-1)}$ locally uniformly at $x$.

The following key theorem can be proved with the aid of Theorem 20.1. For details of proof and further developments see MichalHyers [4].

THEOREM 20.2. Let $q$ be any chosen point of the coordinate domain $\Sigma$ of an allowable $k^{(n+2)}$ coordinate system $x(P)$ in which the above conditions I-IV are satisfied by the linear connection $\Gamma\left(x, \xi_{1}, \xi_{2}\right)$. Then there 
exists a constant $d>0$ and a function $h(p, x)$ of class $C^{(n)}$ uniformly on $E^{2}\left((q)_{2 d}\right)$ such that, for any choice of $p$ in $E\left((q)_{d}\right)$, the transformation $y=h(p, x)$ with inverse $x=\mu(y, p)$ is of class $k^{(n)}$ for $x \in E\left((p)_{d}\right)$ and defines a normal coordinate system $y(P)$ with center $P_{0}=P(p)$.

An important further use of normal coordinates will be made in the study of general projective geometry, general Riemannian geometry, and general conformal geometry. Compare the succeeding chapters.

\section{General projective differential geometry}

21. Projective change of connection. The differential equations of the paths in a differentiable manifold with a linear connection and with a coordinate space $E$ that was employed in Chapter IX take the form (16.3) in a general parameter $t$, while in an affine parameter $s$ they take the form (16.4). The equations (16.3) have the advantage that they retain their form under a general transformation of the parameter $t$, while (16.4) retain their form under an affine transformation of the affine parameter $s$.

One of the interesting questions that arises here is the following: what is the most general linear connection on the given differentiable manifold such that the paths determined by this linear connection are the same as the paths determined by the originally given symmetric linear connection? In other words, what is the most general transformation of linear connection (called projective change of connection) that leaves invariant the differential equations (16.3) of the paths? To answer this question it seems necessary to restrict the coordinate space $E$ still further.

The linear transformations in $E$ form a new Banach space $R$ whose norm function may be taken as the modulus of a linear transformation in $E$. In fact $R$ is a normed ring with unit element. The bilinear ring product $L_{1} L_{2}$ is defined by the succession of two linear transformations in $E$, and the unit $I$ of $R$ is defined by the identical transformation in $E$.

We condition the normed ring $R$, and hence indirectly the space $E$, by the following postulate. $\ddagger$

There exists a linear function $[L]$, called a contraction, on $R$ to the real numbers with the properties: (1) $\left[L_{1} L_{2}\right]=\left[L_{2} L_{1}\right]$; (2) $[N(*) \alpha]=N(\alpha)$, where $N(\xi)$ is linear on $E$ to the real numbers.

Definition. A space $E$, whose normed ring $R$ is so conditioned, will be called a space $E$ with a contraction ring $R$.

$\ddagger$ Michal [10]. 
The following theorem answers the question raised above.

THEOREM 21.1. The most general transformation of the linear connection $\Gamma\left(x, \xi_{1}, \xi_{2}\right)$, called a projective change of connection, that preserves the equation (15.3) of parallelism and hence the corresponding equation (16.3) of paths, is given by

$$
\dagger \Gamma\left(x, \xi_{1}, \xi_{2}\right)=\Gamma\left(x, \xi_{1}, \xi_{2}\right)+\phi\left(x, \xi_{1}\right) \xi_{2}+\phi\left(x, \xi_{2}\right) \xi_{1},
$$

where $\phi(x, \xi)$ is any scalar field valued linear form in a contravariant vector $\xi$.

22. Projective curvature form and projectively flat spaces. Let $B\left(x, \xi_{1}, \xi_{2}, \xi_{3}\right)$ be the c.v.f. valued curvature form (see equation (14.4)) based on the symmetric linear connection $\Gamma\left(x, \xi_{1}, \xi_{2}\right)$. Denote by $B\left(x, \xi_{1}, *, \xi_{2}\right)$ the bilinear function of $\xi_{1}$ and $\xi_{2}$ whose values are in the contraction ring $R$ and which corresponds to $B\left(x, \xi_{1}, \xi, \xi_{2}\right)$ when considered as a linear function of $\xi$. Similarly denote by $B\left(x, *, \xi_{1}, \xi_{2}\right)$ the function which has values in $R$ and which is the correspondent of $B\left(x, \xi, \xi_{1}, \xi_{2}\right)$ when considered as a linear function of $\xi$. With the aid of the contraction function $[L]$ we can define

$$
\begin{aligned}
B\left(x, \xi_{1}, \xi_{2}\right) & =\left[B\left(x, \xi_{1}, *, \xi_{2}\right)\right] \\
\beta\left(x, \xi_{1}, \xi_{2}\right) & =\frac{1}{2}\left[B\left(x, *, \xi_{1}, \xi_{2}\right)\right] \\
\nu & =[I],
\end{aligned}
$$

where $I$ is the unit of $R$.

By a simple argument, we find that $B\left(x, \xi_{1}, \xi_{2}\right)$ and $\beta\left(x, \xi_{1}, \xi_{2}\right)$ are scalar field valued bilinear forms $\ddagger$ in the contravariant vectors $\xi_{1}$ and $\xi_{2}$. It follows, therefore, that the form $W\left(x, \xi_{1}, \xi_{2}, \xi_{3}\right)$, called a projective curvature form and defined by

$$
\begin{aligned}
& W\left(x, \xi_{1}, \xi_{2}, \xi_{3}\right)= B\left(x, \xi_{1}, \xi_{2}, \xi_{3}\right)-\frac{2}{\nu+1} \beta\left(x, \xi_{2}, \xi_{3}\right) \xi_{1} \\
&-\frac{1}{\nu-1}\left(B\left(x, \xi_{1}, \xi_{3}\right) \xi_{2}-B\left(x, \xi_{1}, \xi_{2}\right) \xi_{3}\right) \\
&-\frac{2}{\nu^{2}-1}\left(\beta\left(x, \xi_{1}, \xi_{2}\right) \xi_{3}-\beta\left(x, \xi_{1}, \xi_{3}\right) \xi_{2}\right) \\
& \text { for } \nu=[I] \neq-1,+1,
\end{aligned}
$$

$\ddagger$ More generally, if $T\left(x, \xi_{1}, \xi_{2}, \cdots, \xi_{i-1}, \xi_{i}, \xi_{i+1}, \cdots, \xi_{p}\right)$ is a c.v.f. valued multilinear form in the $p$ contravariant vectors $\xi_{1}, \cdots, \xi_{p}$, then $\left[T\left(x, \xi_{1}, \xi_{2}, \cdots\right.\right.$, $\left.\left.\xi_{i-1}, *, \xi_{i+1}, \cdots, \xi_{p}\right)\right]$, the contraction of $T\left(x, \xi_{1}, \cdots, \xi_{p}\right)$, considered as a linear function of $\xi_{i}$, is a scalar field valued multilinear form in the $p-1$ contravariant vectors $\xi_{1}, \cdots, \xi_{i-1}, \xi_{i+1}, \cdots, \xi_{p}$. 
is a c.v.f. valued trilinear form in the contravariant vectors $\xi_{1}, \xi_{2}, \xi_{3}$. The following two theorems justify the terminology projective curvature form.

THEOREM 22.1. The projective curvature form $W\left(x, \xi_{1}, \xi_{2}, \xi_{3}\right)$ for $\nu=[I] \neq-1,+1$ is a projective differential invariant, that is, it remains invariant under a projective change of connection.

A differentiable manifold with a symmetric linear connection will be said to be projectively flat if there exists a projective change of connection yielding a differentiable manifold with a vanishing curvature form.

THEOREM 22.2. Let $H$ be a 3-differentiable manifold with a symmetric linear connection $\Gamma\left(x, \xi_{1}, \xi_{2}\right)$ that satisfies the restrictions laid down in Chapter IX. Further, assume that the coordinate space is a space E with $a$ contraction ring $R$, and that the functions $\Gamma\left(x, *, \xi_{2}\right), \cdots$, $\Gamma\left(x, \xi_{1}, \xi_{2} ; *\right)$ with values in $R$ possess first differentials in $x$. Finally, we restrict ourselves to numerically valued linear functions $l(x)$ on $E$ that can be written as $l(x)=[\alpha, x]$, and assume that $\nu=[I] \neq-1,+1,+2$. Then a necessary and sufficient condition that $H$ be projectively flat (locally) is that the projective curvature form $W\left(x, \xi_{1}, \xi_{2}, \xi_{3}\right)$ vanish locally.

The more difficult part of the proof of this theorem centers around the differential functional equation

$$
\begin{aligned}
\phi\left(x, \xi_{1} ; \xi_{2}\right)= & \phi\left(x, \Gamma\left(x, \xi_{1}, \xi_{2}\right)\right)+\phi\left(x, \xi_{1}\right) \phi\left(x, \xi_{2}\right) \\
& -\frac{1}{\nu-1} B\left(x, \xi_{1}, \xi_{2}\right)+\frac{2}{\nu^{2}-1} \beta\left(x, \xi_{1}, \xi_{2}\right) .
\end{aligned}
$$

Equation (22.3), together with $W\left(x, \xi_{1}, \xi_{2}, \xi_{3}\right)=0$, is shown to be equivalent to a completely integrable abstract Pfaffian differential equation. The existence of a $\phi(x, \xi)$ is then shown with the aid of an existence theorem of Michal and Elconin [1, 3] on abstract Pfaffian differential equations.

\section{General Riemannian differential Geometry}

23. Element of arc length and abstract Christoffel symbols. A differentiable manifold with a linear connection of special interest is a general Riemannian space whose coordinate space $T$ is an abstract normed linear space $E$ with an independently postulated inner product $[x, y]$. See $\$ 19$ of Chapter IX for the definition of a space $E$.

DeFinition. By a general Riemannian space $\dagger$ with coordinates in an $\dagger$ Michal $[11,12]$. See also Michal [6, 7]. 
abstract space $E$ we mean an m-differentiable manifold $H$ with coordinates in a space $T$ such that

(1) $T$ is a space $E$ and $H$ has allowable $k^{(m)}$ coordinate systems; $\dagger$

(2) the differentials of the coordinate transformations possess adjoints with respect to the inner product $[x, y]$ of $E$ at each point of their domains of definition;

(3) there exists a covariant vector field valued linear form, called a metric form, $g(x, \xi)$ in a contravariant vector $\xi$ with the following properties at each point $x$ of the coordinate domain of every allowable coordinate system:

(3a) $g(x, \xi)$ is differentiable in $x$ up to the pth order, where $p<m$, and where the pth differential is continuous in $x$;

(3b) $[\xi, g(x, \xi)]$ is positive definite in $\xi$;

(3c) $g(x, \xi)$ is a solvable linear function of $\xi$ with $G(x, \eta)$, say, as its inverse function;

(3d) $g(x, \xi)$ is self-adjoint (so that $\left[\xi_{1}, g\left(x, \xi_{2}\right)\right]$ is symmetric in $\xi_{1}$ and $\left.\xi_{2}\right)$;

(3e) the adjoint $g_{(3)}^{*}(x, \xi ; \delta x)$ of the differential $g(x, \xi ; \delta x)$ exists and is itself continuously differentiable to the $(p-1)$ st order (clearly $\left.g_{(3)}^{*}(x, \xi ; \delta x)=g_{(3)}^{*}(x, \delta x ; \xi)\right)$;

(4) the element of arc length is defined by

$$
d s=[\delta x, g(x, \delta x)]^{1 / 2} .
$$

The theory of a general Riemannian space we call a general Riemannian differential geometry.

Define the function $\Gamma\left(x, \xi_{1}, \xi_{2}\right)$ by

$$
\Gamma\left(x, \xi_{1}, \xi_{2}\right)=G\left(x, \gamma\left(x, \xi_{1}, \xi_{2}\right)\right)
$$

where $\ddagger$

$$
\gamma\left(x, \xi_{1}, \xi_{2}\right)=\left\{g\left(x, \xi_{1} ; \xi_{2}\right)+g\left(x, \xi_{2} ; \xi_{1}\right)-g_{(3)}^{*}\left(x, \xi_{1} ; \xi_{2}\right)\right\} / 2 .
$$

It can be shown that $\Gamma\left(x, \xi_{1}, \xi_{2}\right)$, so defined, is the component of a symmetric linear connection, called an abstract "Christoffel" symbol (of second kind). We shall call $\gamma\left(x, \xi_{1}, \xi_{2}\right)$ the "Christoffel" symbol of the first kind.

Clearly the adjoint $\Gamma_{(2)}^{*}(x, \eta, \xi)$ exists $\S$ bilinearly in the covariant vector $\eta$ and contravariant vector $\xi$ and is given by

$\dagger$ For their definition see Michal-Hyers [2, 4].

$\ddagger$ See Michal [6, p. 528] where this expression is given for the first time.

\& In a general Riemannian geometry there thus exist two types of "linear connection": one is the abstract Christoffel symbol, and the other is represented by either one of its adjoints. See Michal $[6,11]$. 


$$
\begin{aligned}
\Gamma_{(2)}^{*}(x, \eta, \xi)= & \gamma_{(2)}^{*}(x, G(x, \eta), \xi) \\
= & \left\{g(x, G(x, \eta) ; \xi)+g_{(3)}^{*}(x, \xi ; G(x, \eta))\right. \\
& -g(x, \xi ; G(x, \eta))\} / 2 .
\end{aligned}
$$

Since $\Gamma$ is a symmetric linear connection, we also have

$$
\Gamma_{(3)}^{*}(x, \xi, \eta)=\Gamma_{(2)}^{*}(x, \eta, \xi) .
$$

The transformation law for the abstract Christoffel symbol of the first kind $\gamma\left(\xi_{1}, \xi_{2}\right)$ in the contravariant vectors $\xi_{1}$ and $\xi_{2}$ is found to be

$$
\bar{\gamma}\left(\bar{x}, \bar{\xi}_{1}, \bar{\xi}_{2}\right)=x^{*}\left(\bar{x} ; \gamma\left(x, \xi_{1}, \xi_{2}\right)\right)+x^{*}\left(\bar{x} ; g\left(x, x\left(\bar{x} ; \bar{\xi}_{1} ; \bar{\xi}_{2}\right)\right)\right) .
$$

The transformation laws for the adjoints of the two abstract Christoffel symbols are derived easily from the corresponding transformation laws of the abstract Christoffel symbols. For example,

$$
\bar{\Gamma}_{(2)}^{*}(\bar{x}, \bar{\eta}, \bar{\xi})=x^{*}\left(\bar{x} ; \Gamma_{(2)}^{*}(x, \eta, \xi)\right)+x_{(2)}^{*}(\bar{x} ; \eta ; \xi)
$$

and

$$
\bar{\gamma}_{(2)}^{*}\left(\bar{x}, \bar{\xi}_{1}, \bar{\xi}_{2}\right)=x^{*}\left(\bar{x} ; \gamma_{(2)}^{*}\left(x, \xi_{1}, \xi_{2}\right)\right)+x_{(2)}^{*}\left(\bar{x} ; g\left(x, \xi_{1}\right) ; \bar{\xi}_{2}\right) .
$$

The following fundamental theorem and its important corollary hold good.

TheOREM 23.1. A necessary and sufficient condition that the covariant differential $g(x, \xi \mid \delta x)$ of a general Riemannian metric $g(x, \xi)$ vanish, where

$$
g(x, \xi \mid \delta x)=g(x, \xi ; \delta x)-g(x, L(x, \xi, \delta x))-L_{(2)}^{*}(x, g(x, \xi), \delta x)
$$

is based on any linear connection $L(x, \xi, \delta x)$ with an adjoint $L_{(2)}^{*}(x, \eta, \delta x)$, is that the symmetrized linear connection $S\left(x, \xi_{1}, \xi_{2}\right)$ $=\left\{L\left(x, \xi_{1}, \xi_{2}\right)+L\left(x, \xi_{2}, \xi_{1}\right)\right\} / 2$ be uniquely determined by the metric $g(x, \xi)$ and the torsion form

$$
\Omega\left(x, \xi_{1}, \xi_{2}\right)=\left\{L\left(x, \xi_{1}, \xi_{2}\right)-L\left(x, \xi_{2}, \xi_{1}\right)\right\} / 2
$$

by means of the formula

$$
\begin{aligned}
S\left(x, \xi_{1}, \xi_{2}\right)= & \Gamma\left(x, \xi_{1}, \xi_{2}\right)+\Omega\left(x, \xi_{1}, \xi_{2}\right) \\
& +G\left(x, \Omega_{(3)}^{*}\left(x, \xi_{1}, g\left(x, \xi_{2}\right)\right)\right) \\
& +G\left(x, \Omega_{(3)}^{*}\left(x, \xi_{2}, g\left(x, \xi_{1}\right)\right)\right),
\end{aligned}
$$

where $\Gamma\left(x, \xi_{1}, \xi_{2}\right)$ is the abstract Christoffel symbol of the second kind based on the general Riemannian metric $g(x, \xi)$. 
COROLlaRy. $\dagger A$ necessary and sufficient condition that the covariant differential $g(x, \xi \mid \delta x)$ of a Riemannian metric $g(x, \xi)$ based on a symmetric linear connection $L\left(x, \xi_{1}, \xi_{2}\right)$ vanish is that $L\left(x, \xi_{1}, \xi_{2}\right)$ be the Christoffel symbol of the second kind $\Gamma\left(x, \xi_{1}, \xi_{2}\right)$ based on the given $g(x, \xi)$.

In other words, in a general Riemannian space with metric $g(x, \xi)$, the functional equation

$$
g(x, \xi ; \delta x)=g(x, L(x, \xi, \delta x))+L_{(2)}^{*}(x, g(x, \xi), \delta x)
$$

has a unique symmetric solution given by the Christoffel symbol of second kind $L\left(x, \xi_{1}, \xi_{2}\right)=\Gamma\left(x, \xi_{1}, \xi_{2}\right)$ of the general Riemannian space. Conversely, if $L(x, \xi, \delta x)=\Gamma(x, \xi, \delta x)$, then the metric $g(x, \xi)$ satisfies the abstract Pfaffian differential equation (23.9). $\ddagger$

24. Abstract Riemann-Christoffel curvature form. If we denote the c.v.f. valued curvature form based on the abstract Christoffel symbol $\Gamma\left(x, \xi_{1}, \xi_{2}\right)$ by $B\left(x, \xi_{1}, \xi_{2}, \xi_{3}\right)$, then it is clear from Theorem 14.2 that in general Riemannian geometry successive covariant differentiation is not a commutative operation (except in a locally flat case, that is, when $B\left(x, \xi_{1}, \xi_{2}, \xi_{3}\right)=0$ in each coordinate domain and for arbitrary contravariant vectors $\left.\xi_{1}, \xi_{2}, \xi_{3}\right)$, and in fact

$$
\xi\left(x\left|\delta_{1} x\right| \delta_{2} x\right)-\xi\left(x\left|\delta_{2} x\right| \delta_{1} x\right)=B\left(x, \xi(x), \delta_{1} x, \delta_{2} x\right)
$$

for arbitrary differentiable c.v.f. $\xi(x)$. by

Define the abstract Riemann-Christoffel curvature form $R\left(x, \xi_{1}, \xi_{2}, \xi_{3}\right)$

$$
R\left(x, \xi_{1}, \xi_{2}, \xi_{3}\right)=g\left(x, B\left(x, \xi_{1}, \xi_{2}, \xi_{3}\right)\right) .
$$

Clearly $R\left(x, \xi_{1}, \xi_{2}, \xi_{3}\right)$ is a cov.v.f. valued trilinear form in the contravariant vectors $\xi_{1}, \xi_{2}, \xi_{3}$.

THEOREM 24.1. The abstract Riemann-Christoffel curvature form can be written in the following useful manner:

$$
\begin{aligned}
R\left(x, \xi_{1}, \xi_{2}, \xi_{3}\right)= & \left\{g\left(x, \xi_{2} ; \xi_{1} ; \xi_{3}\right)+g_{(3)}^{*}\left(x, \xi_{1} ; \xi_{3} ; \xi_{2}\right)\right. \\
& \left.-g_{(3)}^{*}\left(x, \xi_{1} ; \xi_{2} ; \xi_{3}\right)-g\left(x, \xi_{3} ; \xi_{1} ; \xi_{2}\right)\right\} / 2 \\
& +\gamma_{(2)}^{*}\left(x, G\left(x, \gamma\left(x, \xi_{1}, \xi_{3}\right)\right), \xi_{2}\right) \\
& -\gamma_{(2)}^{*}\left(x, G\left(x, \gamma\left(x, \xi_{1}, \xi_{2}\right)\right), \xi_{3}\right) .
\end{aligned}
$$

$\dagger$ Michal [6, p. 528].

$\ddagger$ The Pfaffian equation (23.9) is not, as it stands, a total differential equation of the type studied in Michal-Elconin [3]. 
THEOREM 24.2. The following identities are satisfied by the abstract Riemann-Christoffel curvature form

$$
\begin{aligned}
& R\left(x, \xi_{1}, \xi_{2}, \xi_{3}\right)=-R\left(x, \xi_{1}, \xi_{3}, \xi_{2}\right), \\
& R\left(x, \xi_{1}, \xi_{2}, \xi_{3}\right)+R\left(x, \xi_{3}, \xi_{1}, \xi_{2}\right)+R\left(x, \xi_{2}, \xi_{3}, \xi_{1}\right)=0, \\
& R\left(x, \xi_{1}, \xi_{2}, \xi_{3}\right)=-R_{(2)}^{*}\left(x, \xi_{1}, \xi_{2}, \xi_{3}\right), \\
& R\left(x, \xi_{1}, \xi_{2}, \xi_{3}\right)=R_{(3)}^{*}\left(x, \xi_{3}, \xi_{2}, \xi_{1}\right) .
\end{aligned}
$$

25. Geodesics, parallel displacement and abstract normal coordinates. The extremal curves for the generalized calculus of variations problem

$$
\int_{t_{0}}^{t_{1}}\left\{\left[\frac{d x}{d t}, g\left(x, \frac{d x}{d t}\right)\right]\right\}^{1 / 2} d t=\min .
$$

are found by taking a family of curves

$$
\bar{x}(t)=x(t)+\epsilon \alpha(t) \psi, \quad \alpha\left(t_{0}\right)=\alpha\left(t_{1}\right)=0,
$$

where $\alpha(t)$ is a differentiable real function and $\psi$ is a Banach variable. On extending the classical methods in the obvious way we find that the extremal curves, called geodesics, for the length integral (25.1) satisfy the abstract Euler-Lagrange differential equation

$$
\frac{d^{2} x(s)}{d s^{2}}+\Gamma\left(x, \frac{d x}{d s}, \frac{d x}{d s}\right)=0,
$$

where $\Gamma\left(x, \xi_{1}, \xi_{2}\right)$ is the abstract Christoffel symbol of the second kind (defined in (23.1)) and $s$ is the arc length parameter defined by

$$
s=\int_{t_{0}}^{t}\left\{\left[\frac{d x}{d t}, g\left(x, \frac{d x}{d t}\right)\right]\right\}^{1 / 2} d t .
$$

It follows immediately from (25.3) that a geodesic is an autoparallel curve, that is, the tangent vectors $d x / d s$ of a geodesic form a parallel vector field with respect to that geodesic.

With the aid of Theorem 20.2 and the properties of functions of class $C^{(n)}$ locally uniformly at $x$, the following existence theorem on abstract normal coordinate systems in a general Riemannian space can be established.

THEOREM 25.1. Let $\Sigma$ be the coordinate domain of an allowable $k^{(n+2)}$ coordinate system $x(P)$ in a general Riemannian space with metric form $g(x, \xi)$. If $g(x, \xi)$ and $g_{(3)}^{*}\left(x, \xi_{1} ; \xi_{2}\right)$ are, respectively, of class $C^{(n+1)}$ and $C^{(n)}$ locally uniformly at each $x \in \Sigma$, then the hypotheses of Theorem 20.2 are satisfied when we take the linear connection $\Gamma\left(x, \xi_{1}, \xi_{2}\right)$ 
to be the abstract Christoffel symbol (23.1) of our Riemannian space. The conclusions of Theorem 20.2 hold good, then, for the case on hand under the hypotheses of the present theorem.

Since the abstract Christoffel symbol satisfies $\ddagger$ the identity ${ }^{\dagger} \Gamma(y, y, y)=0$ in a neighborhood of the origin of a normal coordinate system $y(P)$, the following results amongst several others can be proved.

COROLlaRy. The metric form $g(x, \xi)$ of a general Riemannian space satisfies the following differential identities in a neighborhood of the origin of a normal coordinate system $y(P)$ :

$$
\operatorname{tg}(y, y ; y)=0, \quad \operatorname{tg}_{(3)}^{*}(y, y ; y)=0 .
$$

26. Riemannian curvature in a general Riemannian space. $\S$ Let $\lambda_{1}$ and $\lambda_{2}$ be the components of two contravariant vectors (directions). The Riemannian curvature $K$ at a point of a general Riemannian space with respect to the "orientation" $\lambda_{1}, \lambda_{2}$ is defined by

$$
K=\frac{\left[\lambda_{1}, R\left(x, \lambda_{2}, \lambda_{2}, \lambda_{1}\right)\right]}{\left[\lambda_{1}, g\left(x, \lambda_{1}\right)\right]\left[\lambda_{2}, g\left(x, \lambda_{2}\right)\right]-\left[\lambda_{1}, g\left(x, \lambda_{2}\right)\right]^{2}},
$$

where $g(x, \xi)$ is the metric form of the general Riemannian space and $R\left(x, \xi_{1}, \xi_{2}, \xi_{3}\right)$ is the corresponding abstract Riemann Christoffel curvature form (see $\$ 24$ ).

The following theorem furnishes an alternative definition of the Riemannian curvature $K$.

THEOREM 26.1. The Gaussian curvature at a point $P$ of the two dimensional geodesic surface $S$ determined by the orientation $\lambda_{1}, \lambda_{2}$ at $P$ is equal to the Riemannian curvature $K$ given in (26.1).

In the proof of this theorem we make special use of Theorem 25.1 on abstract normal coordinates. The first fundamental form of $S$ induced $\|$ in $S$ by the enveloping general Riemannian space is

$$
b_{\alpha \beta}\left(u^{1}, u^{2}\right) d u^{\alpha} d u^{\beta},
$$

where

$$
b_{\alpha \beta}\left(u^{1}, u^{2}\right)=\left[\frac{\partial y}{\partial w^{\beta}}, g\left(y, \frac{\partial y}{\partial u^{\alpha}}\right)\right]=\left[\lambda_{\beta}, g\left(y, \lambda_{\alpha}\right)\right],
$$

$\ddagger$ See Michal-Hyers [3, p. 166].

$\S$ Michal [12].

\| These considerations foreshadow a general theory of spaces immersed in other spaces. 
and $y$ is an abstract normal coordinate of the general Riemannian space with origin at the point $P$. On computing the Gaussian curvature of $S$,

$$
\frac{R_{1221}}{b_{11} b_{22}-b_{12}^{2}},
$$

we find it equal to the $K$ given in (26.1) and computed for abstract normal coordinates $y$. Hence (26.1) holds in a general coordinate system.

THEOREM 26.2. A necessary and sufficient condition that the Riemannian curvature $K(x)$ at a point of a general Riemannian space be independent of the orientation $\lambda_{1}, \lambda_{2}$ is that the metric form $g(x, \xi)$ satisfy the abstract differential equation at that point

(26.3) $R\left(x, \xi_{1}, \xi_{2}, \xi_{3}\right)=K(x)\left\{\left[\xi_{1}, g\left(x, \xi_{2}\right)\right] g\left(x, \xi_{3}\right)-\left[\xi_{1}, g\left(x, \xi_{3}\right)\right] g\left(x, \xi_{2}\right)\right\}$ for arbitrary contravariant vectors $\xi_{1}, \xi_{2}, \xi_{3}$.

27. General Riemannian spaces of constant Riemannian curvature.* If the Riemannian curvature $K$ is the same for all points (locally) and for all orientations, then the general Riemannian space will be referred to as of constant Riemannian curvature (locally).

The existence of general Riemannian spaces with constant Riemannian curvature follows immediately from the following theorem.

THEOREM 27.1. Let $l(\xi)$ be a solvable self-adjoint linear transformation such that $[\xi, l(\xi)]$ is positive definite, and let

$$
g(x, \xi)=\frac{l(\xi)}{\left\{1+\frac{K_{0}}{4}[x, l(x)]\right\}^{2}} .
$$

Then a general Riemannian space with element of arc length based on the special metric form $g(x, \xi)$ given in (27.1) is a general Riemannian space with a constant Riemannian curvature $K_{0}$.

If the Banach coordinate space $E$ of a general Riemannian space of constant Riemannian curvature $K \neq 0$ is taken to be the usual $n$-dimensional euclidean arithmetic space, then the general Riemannian space is locally one of the classical finite dimensional noneuclidean geometries usually associated with the names of Riemann in the case $K>0$ and with those of Bolyai and Lobatschefski in the case $K<0$.

Amongst the many new infinitely many dimensional examples of

* Michal [12]. 
general Riemannian spaces with constant Riemannian curvature, there are two that are of special interest.* One is obtained by taking the Banach coordinate space $E$ to be Hilbert space with $[x, y]$ as the Hilbert space inner product. The other interesting infinitely many dimensional example is obtained by taking $E$ to be the space of real continuous functions on a real interval $(a, b)$ with the integral over $(a, b)$ of the product of two functions as the inner product of these functions, and by taking

$$
d s^{2}=\frac{l_{\alpha}\left(\delta x^{\alpha}\right)^{2}+l_{\alpha \beta} \delta x^{\alpha} \delta x^{\beta}}{\left\{1+\frac{K_{0}}{4}\left(l_{\alpha}\left(x^{\alpha}\right)^{2}+l_{\alpha \beta} x^{\alpha} x^{\beta}\right)\right\}^{2}},
$$

where $l_{\alpha} \neq 0$ and $l_{\alpha \beta}$ are real continuous functions of the real variable $\alpha$ and the real variables $\alpha$ and $\beta$, respectively, and where the Fredholm determinant $D\left[l_{\alpha \beta} / l_{\beta}\right] \neq 0$. In $(27.2)$ we also understand that a repetition of a real variable in a term, once as a subscript and once as a superscript, denotes Riemann integration with respect to that variable over the interval $(a, b)$. See $\$ 4$.

Up to the present, the coordinate space $E$ of our Riemannian geometries was not required to be space $E$ with a contraction ring $\dagger R$. One of the most interesting theorems in all of general differential geometry seems to depend in an essential manner on the fact that the coordinate space is a space $E$ with a contraction ring $R$. The theorem in question is as follows.

THEOREM 27.2. A general Riemannian space that satisfies the following conditions is a space of constant Riemannian curvature:

(1) the coordinate space is a space $E$ with a contraction ring $R$;

(2) the contraction $[I]$ of the unit element $I$ of the normed ring $R$ is different from 1 or 2 ;

(3) the Riemannian curvature at each point is independent of the orientation $\left(\lambda_{1}, \lambda_{2}\right)$.

It is of some importance to observe that the condition $[I]=1$ or 2 does not necessarily entail a finite dimensional Riemannian space. In fact, there exist infinitely many dimensional spaces for which re-

* Michal [12]. We mention here in passing the important subject of general Riemannian geometry with complex Banach coordinates and a Hermitian differential metric. Infinite dimensional spaces of this type (especially those of constant Riemannian curvature) may be useful in a more satisfactory treatment of various physical theories such as the quantum theory-a mere speculation perhaps, but worthy of serious consideration!

$\dagger$ See $\$ 20$ for the definition of such a coordinate space. 
striction (2) of the above theorem is not satisfied. Furthermore, there exist infinitely many dimensional general Riemannian spaces of constant Riemannian curvature for which restrictions (1) and (3) of the above theorem are satisfied but (2) does not hold-there are cases where $[I]=1$ as well as cases where $[I]=2$.

We mention in passing the important subject of general Riemannian spaces of constant Riemannian curvature considered as spaces immersed $\dagger$ in composite abstract euclidean spaces with coordinates $(x, t)$, where $x$ is a Banach variable and $t$ is a real variable.

28. General conformal differential geometry. $\ddagger$ We have already had examples of conformally flat general Riemannian spaces, that is, spaces whose element of arc length $d s$ is the product of a scalar field and the element of arc length of an abstract euclidean space. The spaces whose metric form $g(x, \xi)$ is given by (27.1) are conformally flat.

The theory of conformally equivalent general Riemann spaces that have coordinate spaces $E$ with a contraction ring $R$ centers around a c.v.f. valued trilinear form $C\left(x, \xi_{1}, \xi_{2}, \xi_{3}\right)$ in the contravariant vectors $\xi_{1}, \xi_{2}, \xi_{3}$. We call this form the conformal curvature form. It is a conformal differential invariant, for it retains its form as a functional of $g(x, \xi)$ and its first two differentials under a differentiable conformal transformation

$$
g^{\prime}(x, \xi)=\lambda(x) g(x, \xi), \quad \lambda(x)>0 .
$$

Its definition is as follows:

$$
\begin{aligned}
C\left(x, \xi_{1}, \xi_{2}, \xi_{3}\right)= & B\left(x, \xi_{1}, \xi_{2}, \xi_{3}\right)+\frac{1}{\nu-2}\left\{-R\left(x, \xi_{1}, \xi_{3}\right) \xi_{2}\right. \\
& +R\left(x, \xi_{1}, \xi_{2}\right) \xi_{3}-\left[\xi_{3}, g\left(x, \xi_{1}\right)\right] G\left(x, R\left(x, \xi_{2}\right)\right) \\
& \left.+\left[\xi_{2}, g\left(x, \xi_{1}\right)\right] G\left(x, R\left(x, \xi_{3}\right)\right)\right\} \\
& +\frac{R(x)}{(\nu-1)(\nu-2)}\left\{\left[\xi_{3}, g\left(x, \xi_{1}\right)\right] \xi_{2}-\left[\xi_{2}, g\left(x, \xi_{1}\right)\right] \xi_{3}\right\},
\end{aligned}
$$

where $B\left(x, \xi_{1}, \xi_{2}, \xi_{3}\right)$ is the curvature form based on the abstract Christoffel symbol $\Gamma\left(x, \xi_{1}, \xi_{2}\right)$ and $\ddagger$

$$
\begin{aligned}
& R\left(x, \xi_{1}, \xi_{2}\right)=\left[B\left(x, \xi_{1}, *, \xi_{2}\right)\right]=\left[\xi_{1}, R\left(x, \xi_{2}\right)\right], \\
& R(x)=[G(x, R(x, *))], \quad \nu=[I] \neq 1,2 .
\end{aligned}
$$

$\dagger$ Michal [12].

$\ddagger$ Michal $[11,12]$. 
H. ARNold

\section{BIBLIOGRAPHY}

1. The Theory of Operational Equations and Differential Transformations in Kantorovitch Spaces, California Institute of Technology Thesis, June, 1939.

S. BANACH

1. Thêorie des Opérations Linéaires, 1932.

GARRETT BIRKHOFF

1. Analytical groups, Transactions of this Society, vol. 43 (1938), pp. 61-101.

2. On product integration, Journal of Mathematics and Physics, vol. 16 (1938), pp. 104-132.

F. Conforto

1. Sopra il calcolo differenziale assoluto negli spazi funzionali continui, Annali della Scuola Normale Superiore di Pisa, vol. 2 (1933), pp. 309-324.

\section{ELCONIN}

1. California Institute of Technology Thesis, not yet completed.

G. C. Evans

1. Functionals and Their Applications. Selected Topics, Including Integral Equa-

M. FRECHET tions, American Mathematical Society Colloquium Publications, vol. 51, 1918.

1. La différentielle dans l'analyse générale, Annales Scientifiques de l'École Normale Supérieure, vol. 42 (1925), pp. 293-323.

2. Les Espaces Abstraits, 1928.

3. Sur la notion de différentielle, Journal de Mathématiques Pures et Appliquées, vol. 16 (1937), pp. 233-250.

T. H. Hilde BRandt and L. M. Graves

1. Implicit functions and their differentials in general analysis, 'Transactions of this Society, vol. 29 (1927), pp. 127-153.

F. HAUSDORFF

1. Mengenlehre, 1927.

I. E. HighBERG

1. Polynomials In Abstract Spaces, California Institute of Technology Thesis, June, 1936.

2. A note on abstract polynomials in complex spaces, Journal de Mathématiques Pures et Appliquées, vol. 16 (1937), pp. 307-314.

D. H. Hyers

1. Integrals and Functional Equations in Linear Topological Spaces, California Institute of Technology Thesis, June, 1937.

2. On functional equations in linear topological spaces, Proceedings of the $\mathrm{Na}$ tional Academy of Sciences, vol. 23 (1937), pp. 496-499.

3. A note on linear topological spaces, this Bulletin, vol. 44 (1938), pp. 76-80.

A. KAWAGUCHI

1. The foundation of the theory of displacements, Proceedings of the Imperial Academy of Japan, vol. 9 (1933), pp. 351-354 (this abstract appeared in 1934).

2. The foundation of the theory of displacements, II, Proceedings of the Imperial Academy of Japan, vol. 10 (1934), pp. 45-48.

3. Die differentialgeometrie in den verschiedenen funktionalraumen, I, Vektorialen und Tensorialen, Journal of Hokkaido Imperial University, vol. 3 (1935), pp. 43-106.

L. S. KENNISON

1. A fundamental theorem on one-parameter continuous groups of projective func- 
tional transformations, Proceedings of the National Academy of Sciences, vol. 16 (1930), pp. 607-609.

2. Reflections in function space, this Bulletin, vol. 38 (1932), pp. 131-134.

M. KERNER

1. La différentielle dans l'analyse générale, Annals of Mathematics, (2), vol. 34 (1933), pp. 546-572.

2. Abstract differential geometry, Compositio Mathematica, vol. 4 (1937), pp. 308341.

A. KolmogorofF

1. Zur Normierbarkeit eines topologischen linearen Raumes, Studia Mathematica, vol. 5 (1934), pp. 29-33.

\section{J. LeRAy AND J. Schauder}

1. Topologie et équations fonctionnelles, Annales Scientifiques de l'École Normale Supérieure, vol. 51 (1934), pp. 45-78.

R. S. MARTIN

1. Contributions to the Theory of Functionals, California Institute of Technology Thesis, June, 1932.

A. D. Michal

1. Affinely connected function space manifolds, American Journal of Mathematics, vol. 50 (1928), pp. 473-517.

2. Differential geometries of function space, Proceedings of the National Academy of Sciences, vol. 16 (1930), pp. 88-94.

3. The differential geometry of a continuous infinitude of contravariant functional vectors, Proceedings of the National Academy of Sciences, vol. 16 (1930), pp. 162-164.

4. Projective functional tensors and other allied functionals, Proceedings of the National Academy of Sciences, vol. 16 (1930), pp 165-168.

5. Function space-time manifolds, Proceedings of the National Academy of Sciences, vol. 17 (1931), pp. 217-225.

6. Riemannian differential geometry in abstract spaces, Proceedings of the National Academy of Sciences, vol. 21 (1935), pp. 526-529.

7. Postulates for a linear connection, Annali di Matematica, vol. 15 (1936), pp. 197-220.

8. General tensor analysis, this Bulletin, vol. 43 (1937), pp. 394-401.

9. Abstract covariant vector fields in a general absolute calculus, American Journal of Mathematics, vol. 59 (1937), pp. 306-314.

10. General projective differential geometry, Proceedings of the National Academy of Sciences, vol. 23 (1937), pp. 546-548.

11. Géométrie conforme gênérale, Comptes Rendus de l'Académie des Sciences, vol. 205 (1937), pp. 552-554.

12. General Riemannian differential geometry. To be published elsewhere.

A. D. Michal and V. Elconin

1. Completely integrable differential equations in abstract spaces, Proceedings of the National Academy of Sciences, vol. 21 (1935), pp. 534-536.

2. Differential properties of abstract transformation groups with abstract parameters, American Journal of Mathematics, vol. 59 (1937), pp. 129-143.

3. Completely integrable differential equations in abstract spaces, Acta Mathematica, vol. 68 (1937), pp. 71-107.

A. D. Michal and D. H. Hyers

1. Second order differential equations with two point boundary conditions in general analysis, American Journal of Mathematics, vol. 58 (1936), pp. 646-660. 
2. Differential invariants in a general differential geometry, Proceedings of the National Academy of Sciences, vol. 23 (1937), pp. 590-593.

3. Theory and applications of abstract normal coordinates in a general differential geometry, Annali della Scuola Normale Superiore di Pisa, vol. 7 (1938), pp. 157-176.

4. Differential invariants in a general differential geometry, Mathematische Annalen, vol. 116 (1939), pp. 310-333.

A. D. Michal, I. E. Highberg and A. E. TAylor

1. Abstract Euclidean spaces with independently postulated analytical and geometrical metrics, Annali della Scuola Normale Superiore di Pisa, vol. 6 (1937), pp. 117-148.

A. D. Michal and R. S. Martin

1. Some expansions in vector space, Journal de Mathématiques Pures et Appliquées, vol. 13 (1934), pp. 69-91.

A. D. Michal ANd A. H. Clifford

1. Fonctions analytiques implicites dans des espaces vectoriels abstraits, Comptes Rendus de l'Académie des Sciences, vol. 197 (1933), pp. 735-737.

A. D. Michal and E. W. Paxson

1. Maps of abstract topological spaces in Banach spaces, this Bulletin, vol. 42 (1936), pp. 529-534.

2. Addendum, this Bulletin, vol. 43 (1937), p. 888 .

3. La différentielle dans les espaces linéaires abstraits avec une topologie, Comptes Rendus de l'Académie des Sciences, vol. 202 (1936), pp. 1741-1743.

4. The differential in abstract linear spaces with a topology, Comptes Rendus de la Société des Sciences de Varsovie, vol. 29 (1936), pp. 106-121.

A. D. Michal and T. S. Peterson

1. The invariant theory of functional forms under the group of linear functional transformations of the third kind, Annals of Mathematics, (2), vol. 32 (1931), pp. 431-450.

A. D. Michal and L. S. Kennison

1. Quadratic functional forms in a composite range, Proceedings of the National Academy of Sciences, vol. 16 (1930), pp. 617-619.

G. C. MoisIL

1. La Mécanique Analytique des Systèmes Continus, Bucarest Thesis, 1929 (appeared in print in 1930).

T. S. Peterson

1. The analogue of Weyl's conformal curvature tensor in a Michal functional geometry, Annali di Matematica, vol. 13 (1934), pp. 55-62.

F. RIESZ

1. Über lineare Funktionalgleichungen, Acta Mathematica, vol. 41 (1918), pp. 71-98.

E. W. Paxson

1. Analysis in Linear Topological Spaces, California Institute of Technology Thesis, June, 1937.

M. H. STONE

1. Linear Transformations in Hilbert Spaces and their Applications to Analysis, American Mathematical Society Colloquium Publications, vol. 15, 1932.

A. E. TAYLOR

1. A reduced set of postulates for abstract Hilbert space, this Bulletin, vol. 41 (1935), pp. 439-448 and pp. 847-848.

2. Analytic Functions in General Analysis, California Institute of Technology Thesis, June, 1936. 
3. Analytic functions in general analysis, Annali della Scuola Normale Superiore di Pisa, vol. 6 (1937), pp. 277-292.

4. On the properties of analytic functions in abstract spaces, Mathematische Annalen, vol. 115 (1938), pp. 466-484.

A. E. TAYLOR AND I. E. HighberG

1. An independent set of postulates for abstract linear spaces, Comptes Rendus de la Société des Sciences de Varsovie, vol. 28 (1935), pp. 136-142.

2. On postulate systems for normed vector spaces, Comptes Rendus de la Société des Sciences de Varsovie, vol. 28 (1935), pp. 142-153.

H. P. Thielman

1. On the invariance of a generalized Gramian in a Riemannian function space, American Journal of Mathematics, vol. 56 (1934), pp. 438-444.

A. TychonofF

1. Ein Fixpunktsatz, Mathematische Annalen, vol. 111 (1935), pp. 767-776.

D. VAN DANTZIG

1. Ricci calculus and functional analysis, Proceedings, Koninklijke Akademie van Wetenschappen, Amsterdam, vol. 39 (1936), pp. 785-794.

\section{VOLTERRA}

1. Theory of Functionals, 1930.

V. VOLTERRA AND J. PERìs

1. Théorie Générale des Fonctionnelles, 1937.

J. von NeumanN

1. Zur Algebra der Funktionaloperationen und Theorie der normalen Operatoren, Mathematische Annalen, vol. 102 (1929-1930), pp. 370-427.

2. On complete topological spaces, Transactions of this Society, vol. 37 (1935), pp. 1-20.

N. WIENER

1. Note on a paper of M. Banach, Fundamenta Mathematicae, vol. 4 (1923), pp. 136-143.

\section{Added October, 1938}

G. D. BIRKHOFF

1. Fifty years of American mathematics, Addresses, American Mathematical Society Semicentennial Publications, vol. 2, 1938, pp. 270-315.

T. Y. THOMAS

1. Recent trends in geometry, Addresses, American Mathematical Society Semicentennial Publications, vol. 2, 1938, pp. 98-135.

A. E. TAYLOR

5. Additions to the theory of polynomials in normed linear spaces, Tôhoku Mathematical Journal, vol. 44 (1938), pp. 302-318.

6. Linear operations which depend analytically on a parameter, Annals of Mathematics, (2), vol. 39 (1938), pp. 574-593.

R. POTIER

1. Sur les espaces abstraits a connexion affine, Comptes Rendus de l'Académie des Sciences, vol. 206 (1938), pp. 1868-1870.

2. Sur les espaces a connexion affine et les espaces Riemanniens généralisés, Comptes Rendus de l'Académie des Sciences, vol. 207 (1938), pp. 27-29.

A. D. MichaL

13. Differential calculus in linear topological spaces, Proceedings of the National Academy of Sciences, vol. 24 (1938), pp. 340-342. 